\title{
The influence of social power on neural responses to emotional conflict
}

\author{
Xueling Ma ${ }^{1}$, Entao Zhang ${ }^{\text {Corresp. } 2,3}$ \\ 1 colloege of psychology Liaoning Normal University, Dalian, China \\ 2 Henan University, Institute of Cognition, Brain and Health, Kaifeng, China \\ 3 Henan University, Institute of Psychology and Behavior, Kaifeng, China \\ Corresponding Author: Entao Zhang \\ Email address: 10030094@vip.henu.edu.cn
}

Background: Major power theories assume that social power can play an important role in an individual's goal-related behaviors. However, the specific psychological mechanisms through which this occurs remain unclear. Some studies suggested that having power enhanced individuals' goal-related behaviors, by contrast, other studies suggested that low-power individuals were associated with a greater performance in goal-directed task. We were particularly interested in how social power changes individuals' goal-related behaviors during an emotional face-word Stroop task.

Method: Social power was primed by asking participants to recall a past situation in which they were in a position of power (high-power individuals), or a situation in which they were lacking power (low-power individuals). Afterward, participants were asked to complete an emotional face-word Stroop task. In the task, words representing specific emotions were written in a prominent red color across a face, and these words and facial expressions were either congruent or incongruent. The participant's task was to judge the emotion of the face while ignoring the red emotional words.

Results: Our behavioral data showed that these individuals displayed faster reaction time and better accuracy in the congruent conditions, slower reaction time for fearful faces and worse accuracy for happy faces in both incongruent and congruent conditions. The event-related potential analyses showed that, compared with low-power individuals, high-power individuals showed greater P1 amplitudes when faced with emotional stimuli (both incongruent and congruent conditions), indicating that power affects individuals' attention in the early sensory processing of emotional stimuli. For the N170 component, lowpower individuals showed more negative amplitudes when facing emotional stimuli, indicated that lowpower individuals paid more attentions to the construct information of emotional stimuli. For the N450 component, compared with congruent conditions, incongruent conditions elicited more negative amplitudes for both high- and low-power individuals. More importantly, fearful faces provoked enhanced P1 amplitudes in the incongruent condition than in the congruent condition only for low-power individuals, while, happy faces elicited larger P1 amplitudes in the congruent condition than in the incongruent condition only for high-power individuals. The findings suggested that during the initial stage of stimuli processing low-power individuals are more sensitive to negative stimuli than high-power individuals.

Conclusion: These findings provided electrophysiological evidence that the differences in the emotional conflict process between high- and low-power individuals mainly lies in the early processing stages of emotional information. Furthermore, evidence from P1 and N170 showed that there was also a redistribution of attentional resources in low-power individuals.

Peer) reviewing PDF | (2020:09:52906:2:0:CHECK 7 Mar 2021) 
$7 \quad$ Correspondence concerning this article should be addressed to Entao Zhang, Ph.D., Department 8 of Psychology, Henan University, Minglun Street, no.85, Kaifeng 475001, Henan Province, 9 China
The influence of social power on neural responses to emotional conflict

$$
\text { Xueling } \mathrm{Ma}^{1} \text {, Entao Zhang }{ }^{2,3 *}
$$

${ }^{1}$ College of Psychology Liaoning Normal University, Dalian, China

${ }^{2}$ Institute of Cognition, Brain and Health, Henan University, Kaifeng, China

${ }^{3}$ Institute of Psychology and Behavior, Henan University, Kaifeng, China

\section{Email: 10030094@vip.henu.edu.cn}

1 Phone: 86-0371-23881810

\section{ABSTRACT}

Background: Major power theories assume that social power can play an important role in an individual's goal-related behaviors. However, the specific psychological mechanisms through which this occurs remain unclear. Some studies suggested that having power enhanced individuals' goal-related behaviors, by contrast, other studies suggested that low-power individuals were associated with a greater performance in goal-directed task. We were particularly interested in how social power changes individuals' goal-related behaviors during an emotional face-word Stroop task.

Method: Social power was primed by asking participants to recall a past situation in which they 
21 were in a position of power (high-power individuals), or a situation in which they were lacking

22 power (low-power individuals). Afterward, participants were asked to complete an emotional

23 face-word Stroop task. In the task, words representing specific emotions were written in a

24 prominent red color across a face, and these words and facial expressions were either congruent

25 or incongruent. The participant's task was to judge the emotion of the face while ignoring the red

26 emotional words.

27 Results: Our behavioral data showed that these individuals displayed faster reaction time and

28 better accuracy in the congruent conditions, slower reaction time for fearful faces and worse

29 accuracy for happy faces in both incongruent and congruent conditions. The event-related

30 potential analyses showed that, compared with low-power individuals, high-power individuals

31 showed greater P1 amplitudes when faced with emotional stimuli (both incongruent and

32 congruent conditions), indicating that power affects individuals' attention in the early sensory

33 processing of emotional stimuli. For the N170 component, low-power individuals showed more

34 negative amplitudes when facing emotional stimuli, indicated that low-power individuals paid

35 more attentions to the construct information of emotional stimuli. For the N450 component,

36 compared with congruent conditions, incongruent conditions elicited more negative amplitudes

37 for both high- and low-power individuals. More importantly, fearful faces provoked enhanced P1

38 amplitudes in the incongruent condition than in the congruent condition only for low-power

39 individuals, while, happy faces elicited larger P1 amplitudes in the congruent condition than in

40 the incongruent condition only for high-power individuals. The findings suggested that during 
41 the initial stage of stimuli processing low-power individuals are more sensitive to negative

42 stimuli than high-power individuals.

43 Conclusion: These findings provided electrophysiological evidence that the differences in the

44 emotional conflict process between high- and low-power individuals mainly lies in the early

45 processing stages of emotional information. Furthermore, evidence from P1 and N170 showed

46 that there was also a redistribution of attentional resources in low-power individuals.

47 Keywords Social power, Emotional conflict, Event-related potential, P1

\section{INTRODUCTION}

51 Social power is a basic social phenomenon characterized by influencing people and resources

52 such as material (food, money, or economic opportunity) and social (knowledge, friendship, or

53 decision-making opportunities) (Keltner, Gruenfeld \& Anderson, 2003). Social power appears to

54 facilitate individuals' ability to pursue goals (Schmid, Kleiman \& Amodio, 2015; Yin \& Smith, 55 2019).

56 The Situated Focus Theory of Power argues that power leads to situated behavior driven by 57 the prioritization of salient goals and constructs (Guinote, 2007a). Social power promotes 58 behavioral flexibility and individuals' behavior that is consistent with goals and motivations, 59 their attention focus can vary with changes in different conditions (Guinote 2007a, 2008; 
60 Overbeck \& Park 2006). In a previous study, they observed a higher behavior variability in high-

61 power individuals than low-power individuals (Guinote, Judd, \& Brauer, 2002). Guinote found

62 that, compared to low-power individuals, high-power individuals paid more attention to work

63 (versus social) information on a weekday and to social (versus work) information on a leisure

64 day. In contrast, low-power individuals tend to pay attention to more peripheral and detailed

65 information, irrespective of its relevance (Guinote, 2017) as they are always under threat

66 (Guinote, 2017). In line with this view, previous studies found that high-power individuals were

67 associated with greater goal focus and performance in the presence of distractors on a variety of

68 response conflict tasks (Guinote, 2007a; Smith, Jostmann, Galinsky, \& van Dijk, 2008; Schmid,

69 Kleiman \& Amodio, 2015).

In contrast, there is also increasing evidence suggesting that high-power individuals are less accurately in recognizing goal information (Martin, Slessor, Allen, Phillips, \& Darling, 2012; Uskul, Paulmann, \& Weick, 2016). For example, a recent study has shown that, in cognitive tasks where participants discriminated sets of objects based on shape, texture, and size, highpower participants performed worse than low-power participants (Weick et al. 2010). More recently, Uskul et al. (2016) provided evidence that high-power individuals are less accurately than low-power individuals when tested on recognition of emotional voices. Thus, the effects of power on information processing are complex and seem to vary in a task dependent manner (Hall et al. 2015).

In the present study, we used the emotional-Stroop task to explore how power affects 
80

81

82

83

84

individuals' goal-related behavior. The emotional-Stroop task is often used by provoking interference through the semantic incompatibility between emotional faces and emotional words, and has been employed in many studies to investigate emotional conflict (Hu et al., 2019; Stickel et al., 2019; Chechko et al. 2012; Chechko et al., 2013), the basic conclusions are that the reaction time for the incongruent condition is significantly higher than the congruent condition, while the accuracy for the incongruent condition is significantly lower than the congruent condition(Zhu et al., 2010). This indicated that in the emotional Stroop task, emotional faces will affect individuals' attention. According to the relevant studies, the emotional-Stroop effect has been shown to activate certain brain mechanisms and to evoke multiple electroencephalography (EEG) components. For example, Shen et al., (2013) found that the N170 was mainly associated with conflicts. Meeren, van Heijnsbergen \& de Gelder (2005) found that when judging emotional faces, the body expression under the congruent condition can produced a larger P1 component in the early stage of perception than the incongruent body expression. And researchers believed that the N450 component may be related to an individual's conflict detection processing in the emotional conflict task (Xue et al., 2015).

Previous studies have examined the relationship between power and attention to others' emotions. However, we were interested in how high- and low-power affects individuals' attention to emotions in incongruent and congruent conditions. The current study took eventrelated potentials (ERPs) to provide accurate information about the time progress of the brain. According to relevant research results and the purpose of this study (Shen et al., 2013; Meeren, 
100

101

102

103

104

van Heijnsbergen \& de Gelder, 2005; Xue et al., 2015), the average amplitude of components of P1 (120-170ms), N170 (150-180ms) and N450 (350-450ms) were analyzed. With reference to past studies (Luo et al., 2010; Tillman \& Wiens, 2011), P7 and P8 electrode points were selected for P1 and N170 analysis. The electrode points of F3, F4, Fz, C3, C4, Cz, CP1, and CP2 were selected for N450 analysis.

To summarize, the present study conducted an emotional conflict task to explore the attention bias in high- and low-power individuals. We hypothesized that (1) N170 amplitude in response to congruent conditions would be larger in the low-power individuals than in the high-power individuals at the P7 electrode; (2) P1 amplitude in response to congruent conditions would be larger in the low-power individuals than in the high-power individuals at the P8 electrode; (3) N450 amplitude in response to congruent conditions would not significantly different between low-power individuals and high-power individuals.

\section{METHOD}

\section{Ethics statement}

Data collection conformed to the Declaration of Helsinki and had been approved by the local Ethics Committee of Henan University (the approval number: HUSOM 2017-217), and all participants signed written informed consent before the experiment.

\section{Participants}

G*Power 3.1 was used to estimate the sample size. For a 2 (social power: high vs. low) $\times 2$ (emotional conflict: incongruent vs. congruent) two-factor mixed design, the significance level $\alpha$ 
$120=0.05$, statistical test power $1-b=0.8$, a moderate effect sizeCohen's $d=0.25$, each group

121 required 17 participants, the total sample size were 34 . In order to improve the statistical test, we

122 still have chosen 20 participants in each group.

12340 college students participated in this experiment. Two participants' data were rejected due to

124 intensive head movements during EEG recording. Finally, 38 participants' data were included

125 (mean age $=21.4$ years, $S D=1.23$ years, 19 males $)$ in this study, 19 each in the high-power $(10$

126 male) and low-power groups (9 male). All participants reported normal or corrected-to-normal

127 vision, right-handed, and had no color vision deficiency or color blindness. Also, they reported

128 no history of affective disorder and were free of any psychiatric medication. Each participant

129 received 50 yuan after an hour of the experiment.

130

131 Apparatus and stimuli

132 Facial images were selected from the Chinese Affective Picture System (CAPS), consisting of 16

133 "Happy" facial images (five females and five males) and 16 "Fear" facial images (five females

and five males). They were cropped faces in a grayscale color. According to the literature (Zhu et

al., 2010), the two Chinese words “愉快” (means “happy”) and “恐惧”(means “fear”) were

written in red on each facial pictures. Finally, we got 64 facial pictures as experimental stimuli.

The incongruent condition was when the facial expression was incongruent with the red Chinese

characters; the reverse was the congruent condition. Among them, 32 images represented the congruent condition and 32 the incongruent condition. The emotional faces were placed in the 
$141 \times 1^{\circ}$ (vertical). The size of face was $9.17 \mathrm{~cm}$ (horizontal) $\times 10.28 \mathrm{~cm}($ vertical).

\section{Experimental procedures}

144 Participants were randomly assigned to either a high-power individuals or low-power individuals.

145 Following the power manipulation, participants performed the emotional-Stroop task and then

146 completed a questionnaire assessing the power manipulation. Data were collected as previously

147 described in Ma et al., (2019), specifically in power manipulation and electroencephalography

148 recordings.

149 Power manipulation

150 Power was manipulated through a retrospective priming procedure (Galinsky, Gruenfeld, \& 151 Magee, 2003; Uskul, Paulmann \& Weick, 2016; Hogeveen, Inzlicht \& Obhi, 2014). In a 152 standardized way, the participants were asked to recall and describe a particular incident in 153 which they had power over another individual (high power prime) or someone else had power 154 over them (low power prime) during the previous week's events and, whether this experience was positive or negative. Emotional-Stroop task. The experimental procedure was controlled

by E-Prime (Psychology Software Tools, Inc., Pittsburgh, PA), this procedure consisted of 4 blocks, and every block consisted of 80 trials, with an equal proportion of each condition. Each trial began with the presentation of a fixation for $400 \mathrm{~ms}$. After a random inter stimulus interval 159 (ISI) between 400 and $600 \mathrm{~ms}$, the stimulus was presented for 1000ms, and finally an inter stimulus interval (ISI) was presented for 1800-2300ms. To avoid priming effects, direct repetitions of the same emotion-word-distractor combination were avoided. The participant's 
162

163

164

165

166

167

168

169

170

171

172

173

174

175

176

177

178

179

180

181

182

task was to rapidly and accurately judge the emotion of the face ("fear" or "happy"), while ignoring the red emotional words (“恐惧” or “愉快”) written on the nose of the faces, by pressing the " 1 " key with their right index finger (happy faces) or the " 2 " key with their right middle finger (fearful faces) (Fig 1).

Insert Fig 1 about here

\section{Manipulation checks}

After the Stroop task was completed, participants were asked to finish a questionnaire which contained two items ( "Now I feel I have a great sense of power" and "Now I feel my wishes don't matter" (reverse scoring)) to complete the power manipulation check (Kraus, Chen \& Keltner, 2011), responses were made using 7-point scales(from 1= "strongly disagree" to 7=

“strongly agree")( $\alpha=0.89)$. Finally, we used The Cognitive Emotion Regulation Questionnaire (CERQ) which contained 36-items to appraise participants' self-control ability (Garnefski, Kraaij \& Spinhoven, 2001).

\section{Electroencephalography recordings and analysis}

The electroencephalogram (EEG) was recorded from 32 scalp sites using electrodes mounted on an $\mathrm{Ag} / \mathrm{AgCl}$ cap (Brain Product), EEG signal was recorded from electrodes arranged according to the standard 10-20 system. Electrodes were placed above and below on the right eye to record the vertical EOG (VEOG). All inter-electrode impedance was maintained below $5 \mathrm{k} \Omega$. The EEG 
183 184 185 186 187 188 189 190

\section{Results of behavioral tests}

\section{RESULTS}

\section{Manipulation checks}

and EOG were amplified using a $0.01-30 \mathrm{~Hz}$ bandpass and continuously sampled at $500 \mathrm{~Hz}$. In the off-line analysis, the epoch of the analysis was from $200 \mathrm{~ms}$ pre-stimulus to $1000 \mathrm{~ms}$ poststimulus. In the experiment, the number of overlapping trials per participant under each stimulus condition was more than 60 times. The analysis was performed using social power (high vs. low) $\times$ emotional conflict (incongruent vs. congruent) $\times$ electrode three-factors repeated-measures ANOVA. In order to understand the results better, the data for the different conditions were analyzed separately: (1) all the faces: incongruent conditions vs congruent conditions; (2) only fearful faces: incongruent conditions vs congruent conditions; and (3) only happy faces: incongruent conditions vs congruent conditions. $P$ values were corrected by Greenhouse-Geisser correction. Post hoc analyses were conducted using Bonferroni corrected $t$-tests.

An ANOVA testing the power manipulation was significant, high-power individuals $(M=4.74$, $S D=0.98)$ felt significantly more powerful than low-power individuals $(M=4.03, S D=0.94)$, $t(36)=-2.29, p=0.028, d=0.74$, which indicated that the power manipulation was effective.

An ANOVA testing the effect of participants' self-control ability, there was no significant differences were found between high and low-power individuals $(t(36)=-0.99, p=0.32)$. 
203 We used PP graph and histogram to check normal distribution. The results suggested that our 204 data basically conforms to the normal distribution. A mixed-model ANOVA with average 205 reaction time as the dependent variable, power as the between-subjects factor, and emotional conflict and emotion as within-subject factors. The main effect of power was not significant, $F(1$, $36)=0.818, p>0.05, \eta 2=0.022$. There was a significant main effect of emotional conflict $(F(1$, $36)=54.238 . p<0.001, \eta 2=0.601)$, reflecting that reaction times in the congruent condition $(M$ $=667, S E=8)$ were significantly faster than in the incongruent condition $(M=697, S E=9)$. There was also a significant effect of emotion $(F(1,36)=4.473, p=0.04, \eta 2=0.11)$, showed that the fearful faces $(M=691, S E=9)$ processed more slowly than happy faces $(M=673, S E=$ $21210)$.

For accuracy rate analyses, the three-way mixed-model ANOVA revealed that the main effect of power was not significant, $F(1,36)=0.005, p>0.05, \eta 2=0.000$. In addition, we found a significant effect of emotional conflict $(F(1,36)=46.807, p<0.001, \eta 2=0.565)$ and of emotion $(F(1,36)=9.222, p=0.004, \eta 2=0.204)$, indicating that a higher level of correct responses during congruent $(91.2 \%)$ compared to incongruent $(82.1 \%)$ conditions and greater accuracy for fear $(88.4 \%)$ compared to happy $(84.9 \%)$ faces. The interaction effect of emotional conflict $\times$ emotion was significant, $F(1,36)=5.507, p=0.025, \eta 2=0.133$. Bonferroni-corrected pairwise comparison indicated a higher level of correct responses during congruent $(92.3 \%)$ compared to

221 the incongruent conditions $(84.6 \%)(p<0.001)$ in fearful faces and greater accuracy for 222 congruent $(90.1 \%)$ compared to the incongruent conditions $(79.7 \%)$ in happy faces $(p<0.001)$ 
223 (Fig 2). There were no other significant results between the RTs and ACC.

224

225

226

227

228

229

230

231

232

233

234

235

236

237

238

239

240

241

242

243

Insert Fig 2 about here

\section{ERP results}

(1) All the faces: incongruent conditions vs congruent conditions

For the $\mathrm{P} 1$ component, the main effect of power was significant $(F(1,36)=2.937, p=0.095$, $\eta 2=0.075)$, the emotional stimuli for high-power individuals primed $(M=0.137, S E=0.693)$ a more positive amplitude than for low-power individuals $(M=-1.544, S E=0.693)$. The main effect of emotional conflict $(F(1,36)=0.042, p=0.838)$, the interaction of power $\times$ emotional conflict $(F(1,36)=1.884, p=0.178)$, the effect of electrode $(F(1,36)=0.087, p=0.769) \mathrm{did}$ not reach significance.

For the N170 component, the main effect of power was significant, $F(1,36)=3.291, p=$ 0.078, $\eta 2=0.084$, the emotional stimuli for low-power individuals elicited a more negative $\mathrm{N} 170(M=-5.466, S E=0.992)$ than for high-power individuals $(M=-2.922, S E=0.992)$. Main effect of emotional conflict $(F(1,36)=0.191, p=0.665)$, and the interaction of power $\times$ emotional conflict $(F(1,36)=0.386, p=0.538)$ were not significant. Meanwhile, a significant main effect of electrode was observed, $F(1,36)=3.164, p=0.084, \eta 2=0.081$, the largest amplitudes were elicited at the P8 $(-4.757 \mu \mathrm{V})$ electrode site.

For the N450 component, the main effect of power $(F(1,36)=0.047, p=0.829)$ were not 
244 significant, while the main effect of emotional conflict was significant, $F(1,36)=5.915, p=$ $2450.02, \eta 2=0.141$, the amplitude was enhanced in the incongruent condition $(M=3.262, S E=$ $2460.484)$ than in the congruent condition $(M=3.806, S E=0.468)$. The interaction of power $\times$ 247 emotional conflict $(F(1,36)=0.79, p=0.38)$ did not reach significance. Meanwhile, a 248 significant main effect of electrode was observed, $F(7,252)=29.809, p=0.000, \eta 2=0.453$, 249 suggesting that the largest N450 amplitudes on CP2 $(M=6.56 \mu \mathrm{V}, S E=0.55)$ (Fig 3 and Fig 4). 250 251

(2) Only fearful faces: incongruent conditions vs congruent conditions

257 For the P1 component, the main effect of power $(F(1,36)=2.469, p=0.125)$ were not 258 significant, while the main effect of emotional conflict was significant, $F(1,36)=6.557, p=$ $2590.015, \eta 2=0.154$, the amplitude was enhanced in the congruent condition $(M=-0.842, S E=$ $0.489)$ than in the incongruent condition $(M=-0.573, S E=0.512)$. The effect of electrode did not reach significance, $F(1,36)=0.185, p=0.67$. The interaction of power $\times$ emotional conflict was significant, $F(1,36)=4.134, p=0.049, \eta 2=0.103)$. Specific contrasts revealed that the P1 
$265=-1.249 \mu \mathrm{V}, S E=0.724$; congruent conditions: $M=-1.731 \mu \mathrm{V}, S E=0.692$ ) but not for high266 power individuals $(F(1,36)=0.139, p=0.711, \eta 2=0.004$; incongruent conditions: $M=$ $2670.102 \mu \mathrm{V}, S E=0.724$; congruent conditions: $M=0.047 \mu \mathrm{V}, S E=0.692$ ).

268 For the N170 component, the main effect of power was significant, $F(1,36)=3.394, p=$ $2690.074, \eta 2=0.086$, the emotional stimuli for low-power individuals elicited a more negative $270 \mathrm{~N} 170(M=-5.45, S E=1.012)$ than for high-power individuals $(M=-2.813, S E=1.012)$. Main 271 effect of emotional conflict was significant, $F(1,36)=4.187, p=0.048, \eta 2=0.104$, congruent 272 conditions elicited a more negative N170 $(M=-4.27, S E=0.705)$ than incongruent conditions $273(M=-3.992, S E=0.733)$. The interaction of power $\times$ emotional conflict $(F(1,36)=1.447, p=$ 274 0.237) was not significant. Meanwhile, the effect of electrode was not significant, $F(1,36)=$ $2752.024, p=0.163$.

276 For the N450 component, the main effect of power $(F(1,36)=0.166, p=0.686)$ were not 277 significant, while the main effect of emotional conflict was significant, $F(1,36)=7.165, p=$ $2780.011, \eta 2=0.166$, the amplitude was enhanced in the incongruent condition $(M=3.058, S E=$ $2790.49)$ than in the congruent condition $(M=3.76, S E=0.501)$. The interaction of power $\times$ 280 emotional conflict $(F(1,36)=0.816, p=0.372)$ was not significant. Meanwhile, the effect of 281 electrode was significant, $F(7,252)=29.585, p=0.000, \eta 2=0.45$, suggesting that the largest $282 \mathrm{~N} 450$ amplitudes on $\mathrm{CP} 2(M=6.479 \mu \mathrm{V}, S E=0.559)($ Fig 5 and Fig 6). 
(3) Only happy faces: incongruent conditions vs congruent conditions.

290

For the P1 component, the main effect of power was significant $(F(1,36)=3.381, p=0.074$, $\eta 2=0.086)$, the emotional stimuli for high-power individuals primed $(M=0.201, S E=0.692) \mathrm{a}$ more positive amplitude than for low-power individual $(M=-1.599, S E=0.692)$. The main effect of emotional conflict $(F(1,36)=2.784, p=0.104)$, the interaction of power $\times$ emotional conflict $(F(1,36)=0.024, p=0.878)$, the effect of electrode $(F(1,36)=0.024, p=0.878) \mathrm{did}$ not reach significance.

The interaction of power $\times$ emotional conflict $\times$ electrode was significant, $F(1,36)=5.70, p=$ $0.022, \eta 2=0.137)$. Specific contrasts revealed that the P1 amplitude was significantly larger in congruent conditions than in incongruent conditions only for high-power individuals $(F(1,36)=$ 5.203, $p=0.029, \eta 2=0.126$; incongruent conditions: $M=0.111 \mu \mathrm{V}, S E=0.914$; congruent conditions: $M=0.629 \mu \mathrm{V}, S E=0.944)$ but not for low-power individuals $(F(1,36)=0.12, p=$

$0.731, \eta 2=0.003$; incongruent conditions: $M=-1.728 \mu \mathrm{V}, S E=0.914$; congruent conditions: $M$ $=-1.65 \mu \mathrm{V}, S E=0.944)$ at $\mathrm{P} 8$ electrode.

For the N170 component, the main effect of power was significant, $F(1,36)=3.149, p=$ 304 $0.084, \eta 2=0.08$, the emotional stimuli for low-power individuals showed a more negative N70 305 $(M=-5.483, S E=0.978)$ than high-power individuals $(M=-3.029, S E=0.978)$ did. Main effect of emotional conflict $(F(1,36)=1.613, p=0.212)$, and the interaction of power $\times$ 
307

308

309

310

311

312

313

314

315

316

317

318

319

320

321

322

323

324

325

326

327

emotional conflict $(F(1,36)=0.058, p=0.811)$ were not significant. Meanwhile, a significant main effect of electrode was observed, $F(1,36)=4.294, p=0.045, \eta 2=0.107$, the largest amplitudes were elicited at the P8 $(-4.949 \mu \mathrm{V})$ electrode site.

For the N450 component, the main effect of power $(F(1,36)=0.000, p=0.986)$, the main effect of emotional conflict $(F(1,36)=2.307, p=0.138)$, the interaction of power $\times$ emotional conflict $(F(1,36)=0.411, p=0.526)$ did not reach significance. Meanwhile, a significant main effect of electrode was observed, $F(7,252)=28.243, p=0.000, \eta 2=0.44$, the largest amplitudes were elicited on $\mathrm{CP} 2(M=6.64 \mu \mathrm{V}, S E=0.555)$ electrode site (Fig 7).

5

6

To evaluate the strength of the empirical evidence, we also conducted a median analysis and a non-parametric test (Mann-Whitney Test). Specifically, the median analysis and the MannWhitney test suggested that for RT, there was a significant difference in low- and high-power individuals only in incongruent conditions when individuals faced happy faces (see Table1 and Table2). Considering the ERP results, the results supported the difference between low-power and high-power individuals when faced fearful faces on N170 and in all conditions on P1 components (see Table1 and Table2).

\section{DISCUSSION}


328 The goal of this study was to test how power facilitates individuals' attention in congruent and

329 incongruent conditions. In the emotional Stroop task, the participants, reacted more slowly and

330 less accurately to incongruent conditions compared to congruent conditions. Therefore, the

331 present research is certainly consistent with the existing results (Shen et al., 2013; Zhou et al.,

332 2015). There were, however, fewer errors overall in the fear face compared to the happy face,

333 suggesting that when it came to targets, attention was likely facilitated by the negative emotional

334 features of the faces based on which participants were required to make their judgment.

335 However, electrophysiological evidence showed the distinction between high-power and low-

power individuals. Specifically, smaller P1 amplitudes were observed for low-power individuals than high-power individuals. The P1 component is considered to reflect the early phase of sensory and perceptual processing of emotional stimuli (Luo, Feng, He, Wang, \& Luo, 2010; Rellecke, Sommer, \& Schacht, 2012). Thus, we inferred that power affected individuals' attention during the sensory processing in both incongruent and congruent conditions. The experimental results could be suggested that more attentional resources were allocated in the early stage in high-power individuals. This result was consistent with the situated focus theory of power and previous findings, which high-power individuals could alter their attentional focus as

344 a function of the demands of the situation. Regarding the N170 component, the low-power individuals showed a more negative N170 amplitude in both congruent and incongruent conditions, indicating that low-power individuals were more concerned with identifying the

347 features of the emotional stimulus itself than high-power individuals, which attenuated the 
349 related to response conflict processing (Chen et al., 2011). More specifically, N450 component 350 was reported to be associated with response conflict detection and resolution (Coderre, Conklin $351 \&$ van Heuven, 2011). Consistenting with the previous ERP studies, the present study found that 352 amplitudes in the N450 components in response to the incongruent condition were larger than to 353 the congruent condition for all of the individuals (Xue et al., 2013; Xue et al., 2015). A possible 354 reason could be that during the early stages, the high-power individuals were required to devote greater attentional resources in the emotional conflict task. However, as time passed and the participants entered the late component stage, the emotional conflict was gradually resolved with the continuous increase in attentional resources (Zhou et al., 2015). This indicated that attentional resource is one of the major factors influencing emotional conflict. Hence, the attentional resources accumulated during the $\mathrm{P} 1$ component in the high-power individuals had a facilitating effect on conflict processing.

361 In addition, the most important result of this study was that in fearful faces, low-power individuals tended to show larger amplitudes than high-power individuals did, and in happy faces, high-power individuals showed larger amplitudes than low-power individuals in the earlier P1 stage. Some ERP studies have shown evidence for an enhanced P1 component for negative relative to neutral stimuli (Vuilleumier, 2005). Our finding suggested that in the initial stage of negative stimuli processing low-power individuals are more sensitive than high-power individuals. Thus, together with work by Guinote (2017), our study at least demonstrated that social power affected individuals' sensory stage. 
369 There were several limitations requiring discussion. On the one hand, the processing of 370 emotional expressions is not only affected by individual power, but also biased by target power 371 (emotion expresser) (Ratcliff, Bernstein, Cundiff, \& Vescio, 2012a; Ratcliff, Franklin, Nelson, \& 372 Vescio, 2012b). The situated focus theory of power proposed that high-power individuals are 373 supposed to dynamically adapt in a complex, goal-congruent way to the target' s power (Côté et 374 al., 2011; Guinote, 2010). On the other hand, without the neutral (power control) condition, there 375 is reason to doubt that the modulated effect of power on emotional conflict tasks might also be 376 found in the power control condition, which would make the results less convinced.

377

378

\section{CONCLUSION}

In summary, utilizing high temporal-resolution ERP technology, we provided solid evidence to support the view that social power can be an important factor in affecting emotional conflict in the early processing stages of emotional information. Our results suggested that there was a redistribution of attentional resources in low power individuals. The findings above have further enriched the theoretical research on the relationship between social power and attention. Based on these results, we proposed that power effects on individuals' attention are an important goal for future research. Our findings could help to revise and qualify existing theories of how power affects attention.

\section{ADDITIONAL INFORMATION AND DECLARATIONS}

\section{Funding}


390 This study was funded by the National Natural Science Foundation of China (31700952) and the

391 Philosophy and Social Science Foundation of Henan Province, China (2018BJY008).

392

393

Grant Disclosures

394 The following grant information was disclosed by the authors:

395 National Natural Science Foundation of China: 31700952.

396 Philosophy and Social Science Foundation of Henan Province, China: 2018BJY008.

397

398 Competing Interests

399 The authors declare that they have no competing interests.

400

401

Author Contributions

402

403

Xueling Ma designed the experiments, performed the experiments, analyzed the data, prepared

404

figures and/or tables, authored or reviewed drafts of the paper, approved the final draft.

405

Entao Zhang conceived and designed the experiments, contributed reagents/materials/analysis

406 tools, authored or reviewed drafts of the paper, and approved the final draft.

407

408

Human Ethics

409 The following information was supplied relating to ethical approvals (i.e., approving body and 410 any reference numbers): 
411 Data collection conformed to the Declaration of Helsinki and had been approved by the local

412 Ethics Committee of Henan University, and all participants signed written informed consent

413 before the experiment.

414

415 Data Availability

416 The following information was supplied regarding data availability:

417 The raw data has been supplied as Supplemental Dataset Files.

References

Chechko N, Kellermann T, Zvyagintsev M, Augustin M, Schneider F, Habel U. 2012. Brain circuitries involved in semantic interference by demands of emotional and non-emotional distractors. PloS One 7(5):

424 Chechko N, Augustin M, Zvyagintsev M, Schneider F, Habel U, Kellermann T. 2013. Brain circuitries 149(1-3): 136-145 DOI 10.1016/ j.jad.2013.01.013.

427 Chen A, Bailey K, Tiernan BN, West R. 2011. Neural correlates of stimulus and response interference in a 2-1 10.1016/j.ijpsycho.2011.02.012.

430 Coderre E, Conklin K, van Heuven WJ. 2011. Electrophysiological measures of conflict detection and 
431

432

433

434

435

436

437

438

439

440

441

442

resolution in the Stroop task. Brain Research 1413(6): 51-59 DOI 10.1016/j.brainres.2011.07.017.

Côté S, Kraus MW, Cheng BH, Oveis C, van der Löwe I, Lia H, Keltner D. 2011. Social power facilitates the effect of prosocial orientation on empathic accuracy. Journal of Personality and Social Psychology 101: 217-232 DOI 10.1037/a0023171.

Galinsky AD, Gruenfeld DH, Magee J.C. 2003. From power to action. Journal of Personality and Social Psychology 85: 453-466 DOI 10.1037/0022-3514.85.3.453.

Garnefski N, Kraaij V, Spinhoven P. 2001. Negative life events, cognitive emotion regulation, and emotional problems. Personality \& Individual Differences 30(8): 1311-1327 DOI 10.1016/S0191-8869(00)00113-6.

Guinote A, Judd CM, Brauer M. 2002. Effects of power on perceived and objective group variability: evidence that more powerful groups are more variable. J. Pers. Soc. Psychol 82(5):708-21 DOI 10.1037/00223514.82.5.708.

Guinote A. 2007a. Behavior variability and the Situated Focus Theory of Power. European Review of Social Psychology 18: 256-295 DOI 10.1080/10463280701692813.

Guinote A. 2008. Power and affordances: When the situation has more power over powerful than powerless individuals. Journal of Personality and Social Psychology 95: 237-252 DOI 10.1037/ a0012518.

Guinote A. 2010. The situated focus theory of power. In A. Guinote T. Vescio (Eds.), The social psychology of power (pp: 141-176). New York, NY: Guilford Press DOI discovery.ucl.ac.uk/133607/.

Guinote A. 2017. How Power Affects People: Activating, Wanting, and Goal Seeking. Annual Review of Psychology 68(1): 353-381 DOI 10.1146/annurev-psych-010416-044153.

Hall JA, Schmid M M, Latu IM. 2014. The Vertical Dimension of Social Relations and Accurate Interpersonal Perception: A Meta-Analysis. Journal of Nonverbal Behavior 39(2): 131-163 DOI 10.1007/s10919-0140205-1 
453 Hogeveen J, Inzlicht M, Obhi SS. 2014. Power changes how the brain responds to others. Journal of

454

455

456

457

458

459

460

461

462

463

464

465

466

467

468

469

470

471

472

473 Experimental Psychology: General 143: 755-762 DOI 10.1037/a0033477.

Hu YY, Zhu JC, Ge Y, Luo WB, Pu X. 2019. Differences in the emotional conflict task between individuals with high and low social adjustment: an erp study. PLoS ONE 14(6): e0217962- DOI 10.1371/journal.pone.0217962.

Keltner D, Gruenfeld DH, Anderson C. 2003. Power, approach, and inhibition. Psychological Review 110(2): 265-84 DOI 10.1037/0033-295X.110.2.265.

Kraus MW, Chen S, Keltner D. 2011. The power to be me: Power elevates self-concept consistency and authenticity. Journal of Experimental Social Psychology 47(5): 974-980 DOI 10.1016/j.jesp.2011.03.017.

Luo W, Feng W, He W, Wang NY, Luo YJ. 2010. Three stages of facial expression processing: erp study with rapid serial visual presentation. Neuroimage 49(2): 1857-1867 DOI 10.1016/j.neuroimage.2009.09.018.

Martin D, Slessor G, Allen R, Phillips L H, Darling S. 2012. Processing orientation and emotion recognition. Emotion 12: 39-43 DOI 10.1037/a0024775.

Ma XL, Wu K, Zhang ET. 2019. The role of social power in neural responses to others' pain. Frontiers in Psychology 10: 2320 DOI 10.3389/fpsyg.2019.02320.

Meeren HKM, van Heijnsbergen CCRJ, de Gelder B. 2005. Rapid integration of facial expression and emotional body language. Proceedings of the National Academy of Sciences of the United States of America 102(45): 16518-16523 DOI 10.1073/pnas.0507650102.

Overbeck JR, Park B. 2006. Powerful perceivers, powerless objects: Flexibility of powerholders' social attention. Organ Behav Hum Decis Process 99: 227-243 DOI 10.1016/j.obhdp.2005.10.003

Rellecke J, Sommer W, Schacht A. 2012. Does processing of emotional facial expressions depend on intention? 
474

Time-resolved evidence from event-related brain potentials. Biological Psychology 90: 23-32 DOI 10.1016/j.biopsycho.2012.02.002.

Ratcliff NJ, Bernstein MJ, Cundiff JL, Vescio TK. 2012a. Seeing wrath from the top (through stratified lenses): perceivers high in social dominance orientation show superior anger identification for high-status individuals. Journal of Experimental Social Psychology48(6): 1373-1376 DOI 10.1016/j.jesp.2012.05.016.

Ratcliff NJ, Franklin JRG, Nelson AJ, Vescio TK. 2012b. The scorn of status: A bias toward perceiving anger on high-status faces. Social Cognition 30: 631-642 DOI 101521 soco2012305631.

Schmid PC, Kleiman T, Amodio DM. 2015. Power effects on cognitive control: turning conflict into action. Journal of Experimental Psychology: General 144(3): 655-663 DOI 10.1037/xge0000068.

Smith PK, Jostmann NB, Galinsky AD, van Dijk WW. 2008. Lacking power impairs executive functions. Psychological Science 19: 441-447 DOI 10.1111/j.1467-9280.2008.02107.x.

Shen YM, Xue S, Wang KC, Qiu J. 2013. Neural time course of emotional conflict control: an erp study. Neuroence Letters 541: 34-38 DOI 10.1016/j.neulet.2013.02.032.

Stickel S, Eickhoff S, Goecke TW, Schneider F, Quinete NS, Lang J, Habel U, Chechko N. 2019. Cumulative cortisol exposure in the third trimester correlates with postpartum mothers' neural response to emotional interference. Biological psychology 143: 53-61 DOI 10.1016/j.biopsycho.2019.02.008.

Tillman CM, Wiens S. 2011. Behavioral and ERP indices of response conflict in Stroop and flanker tasks. Psychophysiology 48(10): 1405-1411 DOI 10.1111/j.1469-8986.2011.01203.x.

Uskul AK, Paulmann S, Weick M. 2016. Social power and recognition of emotional prosody: High power is associated with lower recognition accuracy than low power. Emotion 16(1):11-15 DOI 10.1037/emo0000110.

Vuilleumier P. 2005. How brains beware: neural mechanisms of emotional attention. Trends in Cognitive Sciences 9(12): 585-594 DOI 10.1016/j.tics.2005.10.011. 
498

499

500

501

502

503

504

505

506

507

508

509

510

511

512

513

514

515

516

517

518

Weick M, Guinote A. 2010. How long will it take? power biases time predictions. Journal of Experimental Social Psychology 46(4): 595-604 DOI 10.1016/j.jesp.2010.03.005.

Xue S, Cui J, Wang K, Zhang S, Qiu J, Luo Y. 2013. Positive emotion modulates cognitive control: an eventrelated potentials study. Scandinavian Journal of Psychology 54(2): 82-88 DOI 10.1111/sjop.12031.

Xue S, Ren G, Kong X, Liu J, Qiu J. 2015. Electrophysiological correlates related to the conflict adaptation effect in an emotional conflict task. Neuroence Letters 584: 219-223 DOI 10.1016/j.neulet.2014.10.019.

Yin Y, Smith PK. 2019. Power and cognitive functioning. Current Opinion in Psychology 33: 95-99 DOI 10.1016/j.copsyc.2019.07.020.

Zhou PY, Yang GC, Nan WZ, Liu X. 2015. The time course of attentional modulation on emotional conflict processing. Cognition \& Emotion 30(4): 621-637 DOI 10.1080/02699931.2015.1020051.

Zhu XR, Zhang HJ, Wu TT, Luo WB, Luo YJ. 2010. Emotional conflict occurs at an early stage: Evidence from the emotional face-word Stroop task. Neuroscience Letters 478(1): 1-4 DOI 10.1016/j.neulet.2010.04.036.

\section{Figure Captions}

Fig 1. (A) Sequence of events in a representative trial of the experiment, and (B) the example stimuli. Participants were asked to identify the faces with fearful or happy expressions that had either "fear" or "happy" written across them. Stimulus material consisted of congruent and incongruent conditions (In the actual experiment, the images were the real 
person pictures from the Chinese Affective Picture System).

520

521

522

523

524

525

526

527

528

529

530

531

532

533

534

535

536

537

538

539

540

541

542

543

544

545

Fig 2. (A) Descriptive statistics for response times (ms) for high-power individuals and (B) for low-power individuals, and (C) accuracy rate (\%) for high-power individuals and (D) for low-power individuals.

Fig 3. (A) The Grand-average $\mathrm{P} 1$ in incongruent conditions at $\mathrm{P} 8$ electrode site for all the faces, and (B) Topographical maps of the P1 for low-power individuals and (C) for high-power individuals in incongruent conditions. (D) The Grand-average P1 in congruent conditions at P8 electrode site for all the faces, and (E) Topographical maps of the P1 for low-power individuals and (F) for high-power individuals in congruent conditions. (G) The Grandaverage N170 in incongruent conditions at P7 electrode site for all the faces, and (H) Topographical maps of the N170 for low-power individuals and (I) for high-power individuals in incongruent conditions. (J) The Grand-average N170 in congruent conditions at P7 electrode site for all the faces, and (K) Topographical maps of the N170 for lowpower individuals and (L) for high-power individuals in congruent conditions.

Fig 4. (A) The Grand-average N450 in low-power individuals at CZ electrode site for all the faces, and (B)Topographical maps of the N450 in incongruent conditions and (C) in congruent conditions for low-power individuals. (D) The Grand-average N450 in highpower individuals at $\mathrm{CZ}$ electrode site for all the faces, and (E) Topographical maps of the N450 in incongruent conditions and (F) in congruent conditions for high-power individuals.

Fig 5. (A) The Grand-average P1 in low-power individuals at P7 electrode site for fearful faces, and (B) Topographical maps of the P1 in incongruent conditions and (C) in congruent conditions for low-power individuals. (D) The Grand-average P1 in high-power individuals at P7 electrode site for fearful faces, and (E) Topographical maps of the P1 in incongruent conditions and (F) in congruent conditions for high-power individuals. (G) The Grandaverage N170 in incongruent conditions at P8 electrode site for fearful faces, and (H) Topographical maps of the N170 for low-power individuals and (I) for high-power individuals in incongruent conditions. (J) The Grand-average N170 in congruent conditions 
546

547

548

549

550

551

552

553

554

555

556

557

558

559

560

561

562

563

564

565

566

567

568

569

at P8 electrode site for fearful faces, and (K) Topographical maps of the N170 for lowpower individuals and (L) for high-power individuals in congruent conditions.

Fig 6. (A) The Grand-average N170 in low-power individuals at P7 electrode site for fearful faces, and (B) Topographical maps of the N170 in incongruent conditions and (C) in congruent conditions for low-power individuals. (D) The Grand-average N170 in highpower individuals at P7 electrode site for fearful faces, and (E) Topographical maps of the N170 in incongruent conditions and (F) in congruent conditions for high-power individuals. (G) The Grand-average N450 in low-power individuals at CZ electrode site for fearful faces, and $(\mathrm{H})$ Topographical maps of the N450 in incongruent conditions and (I) in congruent conditions for low-power individuals. (J) The Grand-average N450 in high-power individuals at $\mathrm{CZ}$ electrode site for fearful faces, and $(\mathrm{K})$ Topographical maps of the N450 in incongruent conditions and (L) in congruent conditions for high-power individuals.

Fig 7. (A) The Grand-average P1 in incongruent conditions at P8 electrode site for happy faces, and (B) Topographical maps of the P1 for low-power individuals and (C) for high-power individuals in incongruent conditions. (D) The Grand-average P1 in congruent conditions at P8 electrode site for happy faces, and (E) Topographical maps of the P1 for low-power individuals and (F) for high-power individuals in congruent conditions. (G) The Grandaverage N170 in incongruent conditions at P8 electrode site for happy faces, and (H) Topographical maps of the N170 for low-power individuals and (I) for high-power individuals in incongruent conditions. (J) The Grand-average N170 in congruent conditions at P8 electrode site for happy faces, and (K) Topographical maps of the N170 for lowpower individuals and (L) for high-power individuals in congruent conditions.

Table 1 The median for all conditions

Table 2 Non-parametric test for all conditions 


\section{Figure 1}

Experimental procedures and the example stimuli

(A) Sequence of events in a representative trial of the experiment, and (B) the example stimuli. Participants were asked to identify the faces with fearful or happy expressions that had either "fear" or "happy" written across them. Stimulus material consisted of congruent and incongruent conditions (In the actual experiment, the images were the real person pictures from the Chinese Affective Picture System). 
(A)
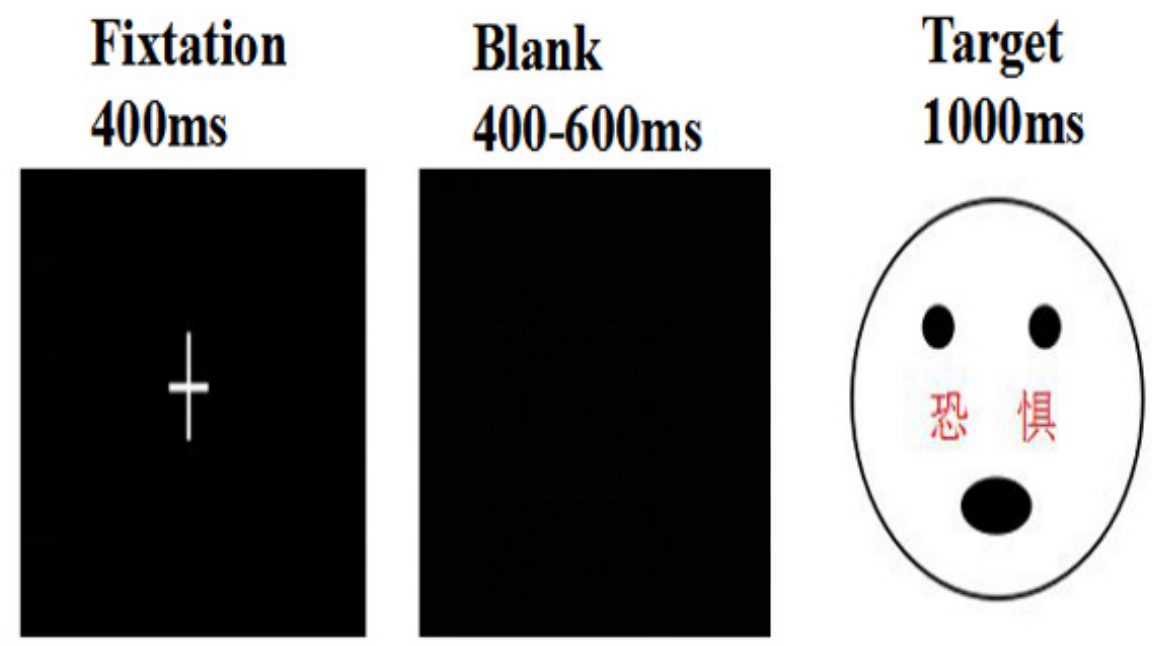

Blank
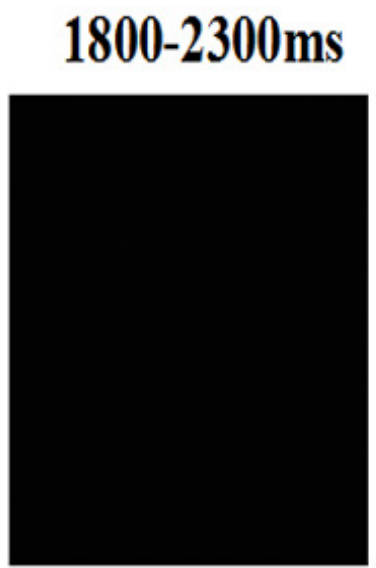

Time

(B)

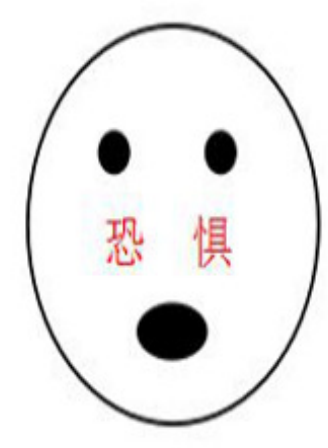

congruent

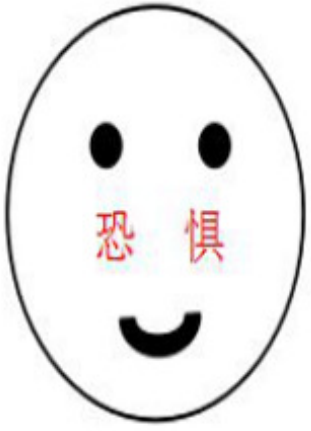

incongruent 
Figure 2

Descriptive statistics for response times (ms) and accuracy rate(\%) for high- and lowpower individuals.

(A) Descriptive statistics for response times (ms) for high-power individuals and (B) for lowpower individuals, and (C) accuracy rate (\%) for high-power individuals and (D) for low-power individuals. 


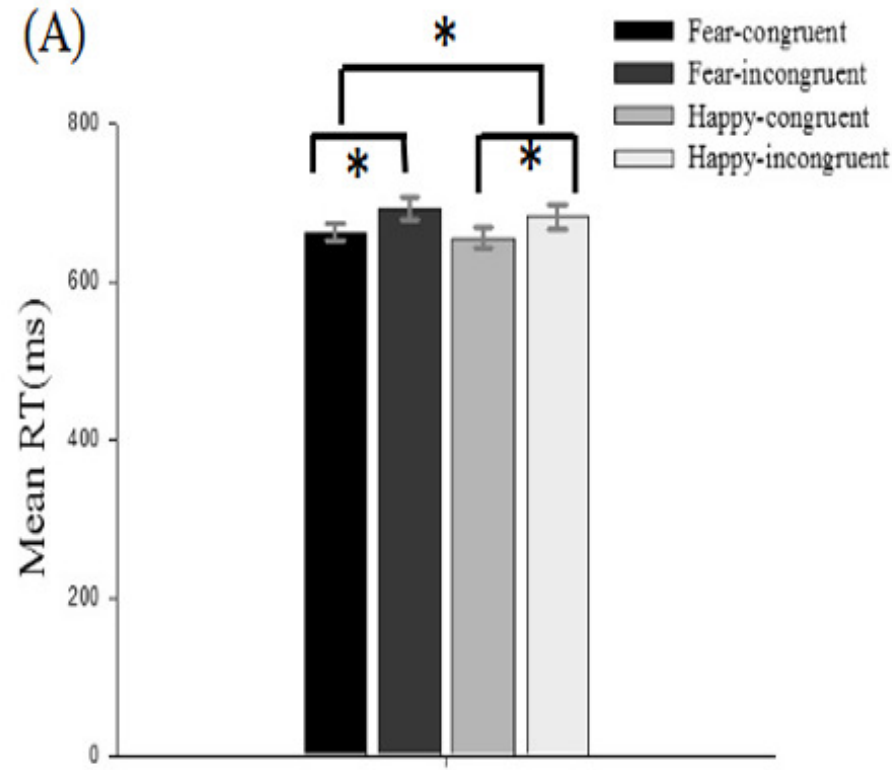

High-power individuals

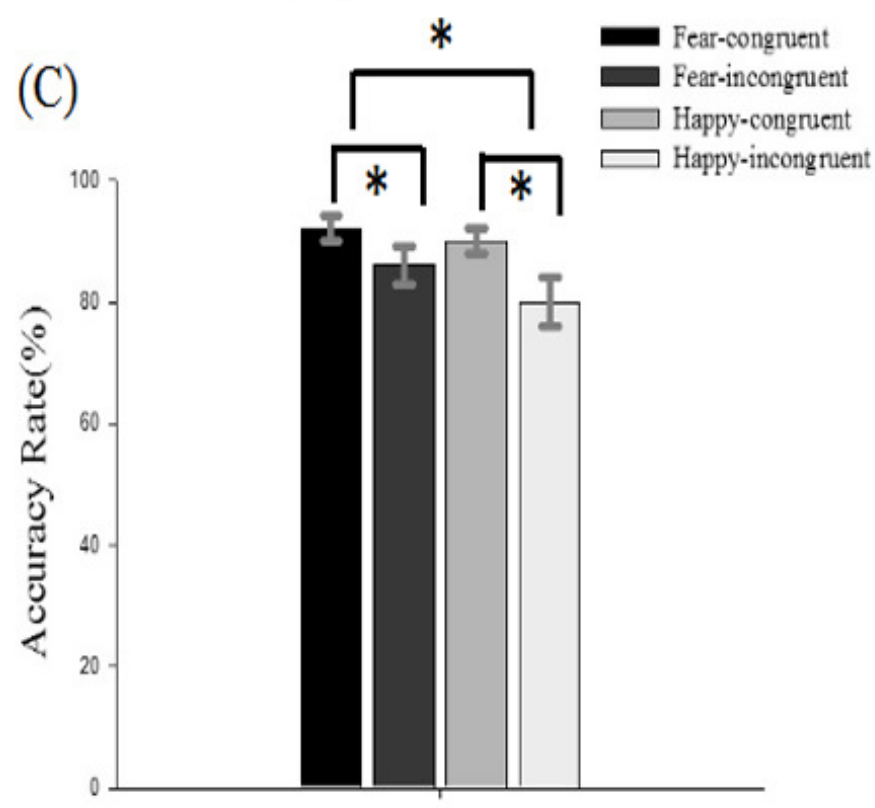

High-power individuals

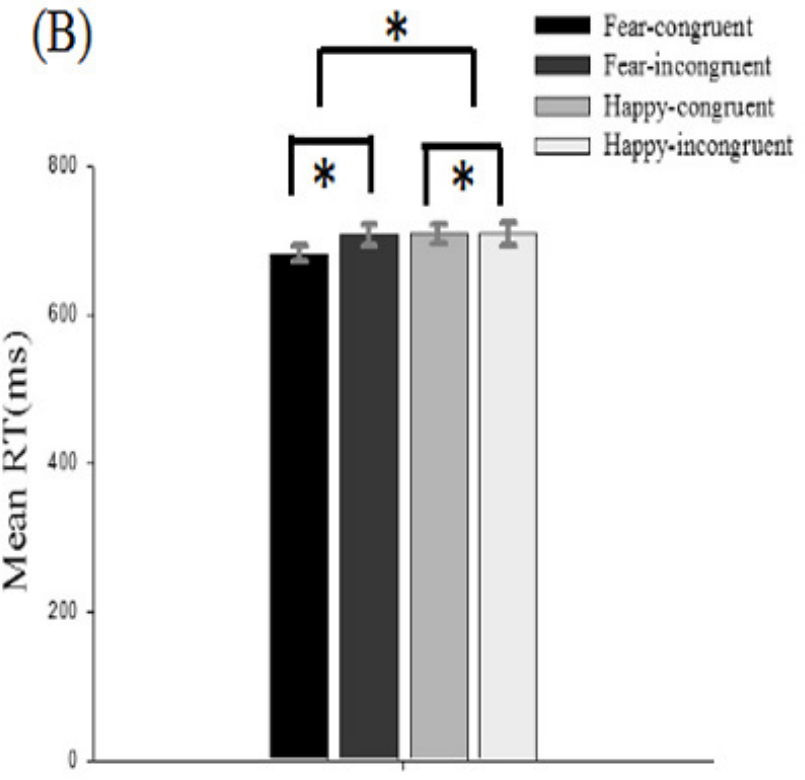

Low-power individuals

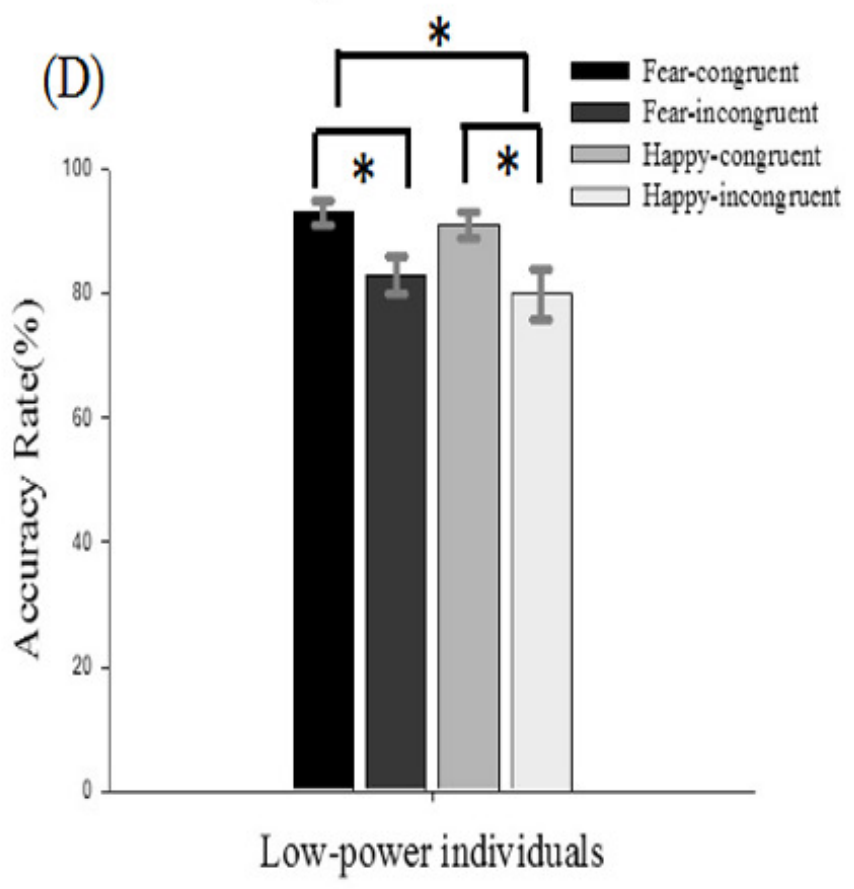




\section{Figure 3}

Waveforms and topographical maps of P1 and N170 components for all the faces.

(A) The Grand-average P1 in incongruent conditions at P8 electrode site for all the faces, and

(B) Topographical maps of the P1 for low-power individuals and (C) for high-power individuals in incongruent conditions. (D) The Grand-average P1 in congruent conditions at P8 electrode site for all the faces, and (E) Topographical maps of the P1 for low-power individuals and (F) for high-power individuals in congruent conditions. (G) The Grand-average N170 in incongruent conditions at P7 electrode site for all the faces, and (H) Topographical maps of the N170 for low-power individuals and (I) for high-power individuals in incongruent conditions. (J) The Grand-average N170 in congruent conditions at P7 electrode site for all the faces, and (K) Topographical maps of the N170 for low-power individuals and (L) for highpower individuals in congruent conditions. 
(A)

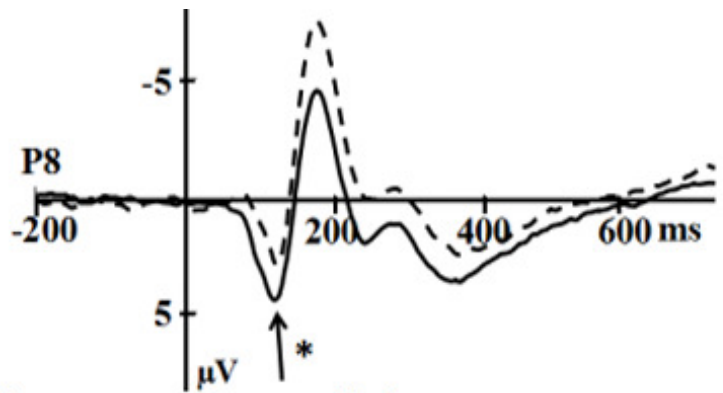

Incongruent conditions

(D)

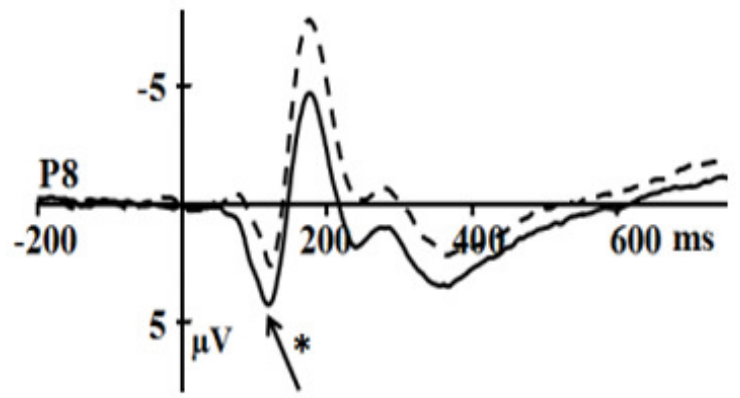

P1(120-170ms)

Low-power individuals High-power individuals

(B)

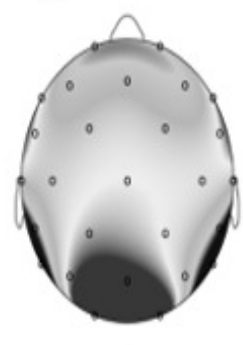

(E)

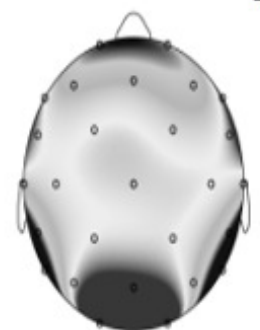

(C)

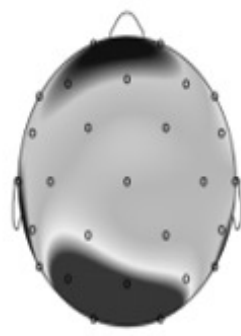

(F)

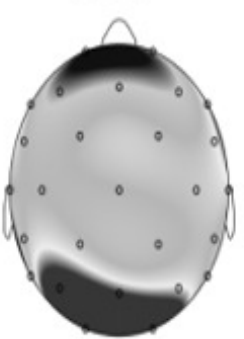

Congruent conditions —High-power individuals

(G)

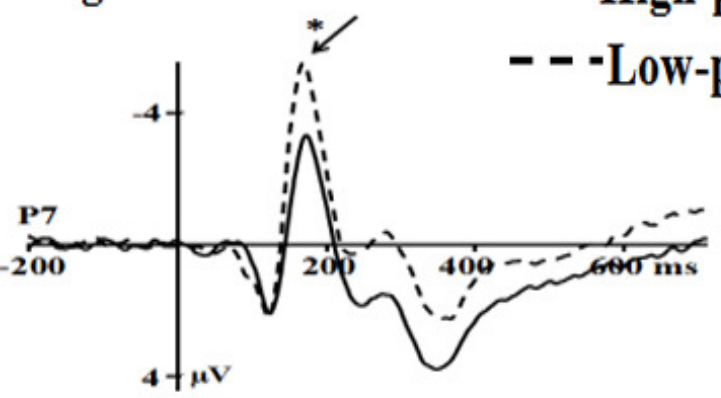

Incongruent conditions

(J)

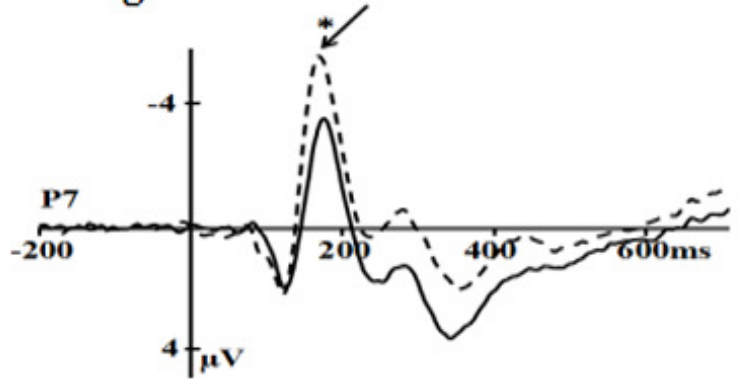

Congruent conditions
(H)

Low-power individuals High-power individuals

(K)

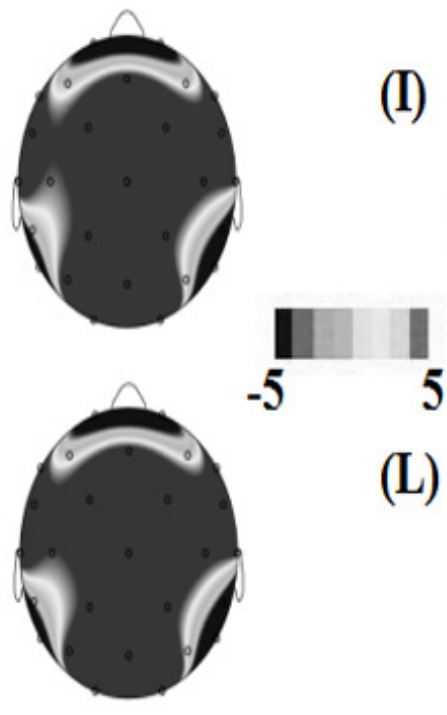

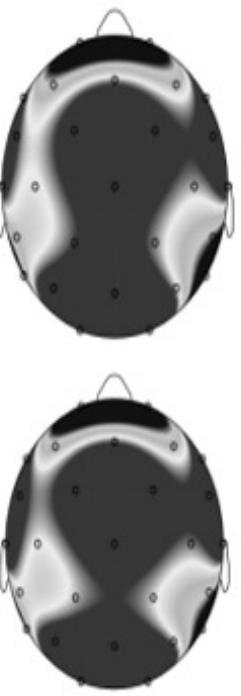




\section{Figure 4}

Waveforms and topographical maps of N450 components for all the faces.

(A) The Grand-average N450 in low-power individuals at CZ electrode site for all the faces, and (B)Topographical maps of the N450 in incongruent conditions and (C) in congruent conditions for low-power individuals. (D) The Grand-average N450 in high-power individuals at CZ electrode site for all the faces, and (E) Topographical maps of the N450 in incongruent conditions and $(F)$ in congruent conditions for high-power individuals. 

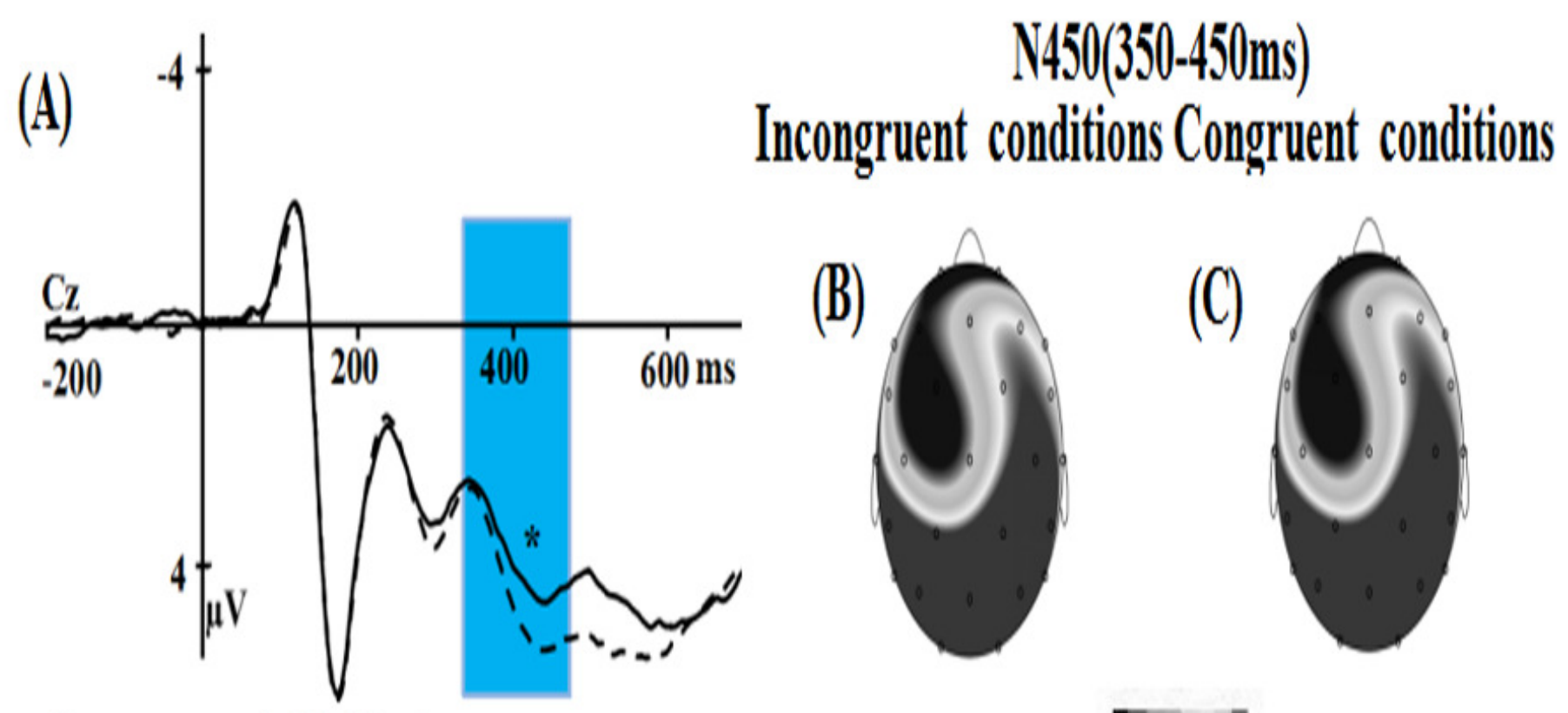

Low-power individuals
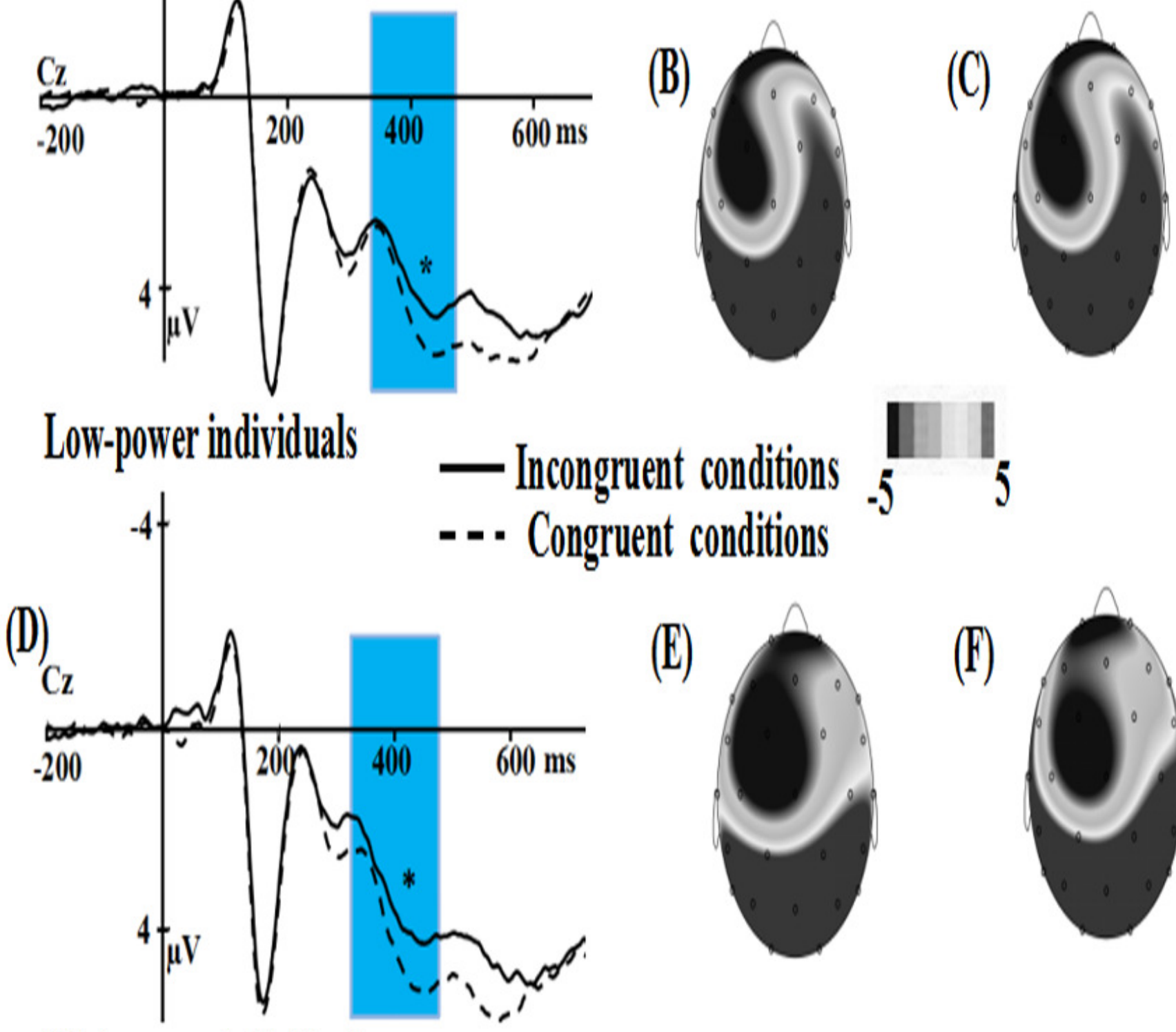

(E)

High-power individuals

\section{- Incongruent conditions}

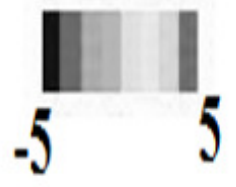

(F)
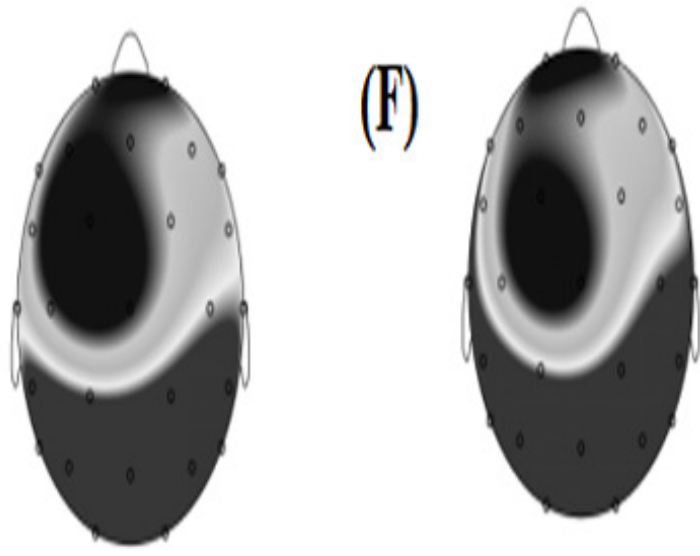


\section{Figure 5}

Waveforms and topographical maps of P1 and N170 components for fear faces.

(A) The Grand-average P1 in low-power individuals at P7 electrode site for fear faces, and (B) Topographical maps of the P1 in incongruent conditions and (C) in congruent conditions for low-power individuals. (D) The Grand-average P1 in high-power individuals at P7 electrode site for fear faces, and (E) Topographical maps of the P1 in incongruent conditions and (F) in congruent conditions for high-power individuals. (G) The Grand-average N170 in incongruent conditions at P8 electrode site for fear faces, and (H) Topographical maps of the N170 for low-power individuals and (I) for high-power individuals in incongruent conditions. (J) The Grand-average N170 in congruent conditions at P8 electrode site for fear faces, and (K) Topographical maps of the N170 for low-power individuals and (L) for high-power individuals in congruent conditions. 
(A)

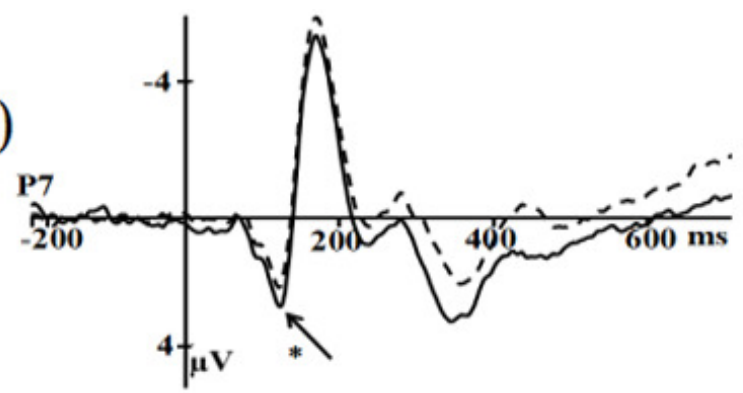

(D)

Low-power individuals - Incongruent conditions

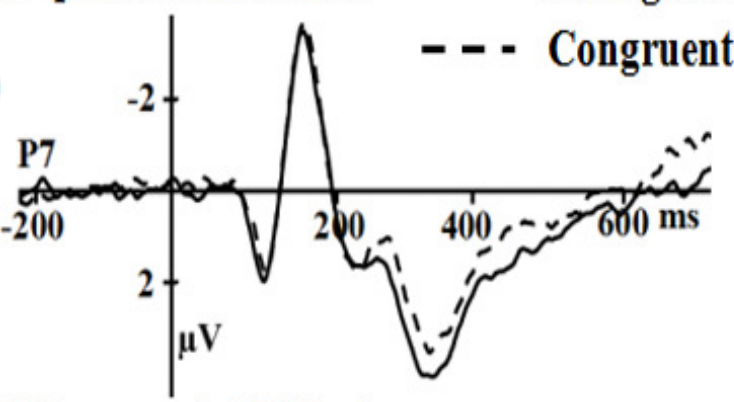

High-power individuals

(G)

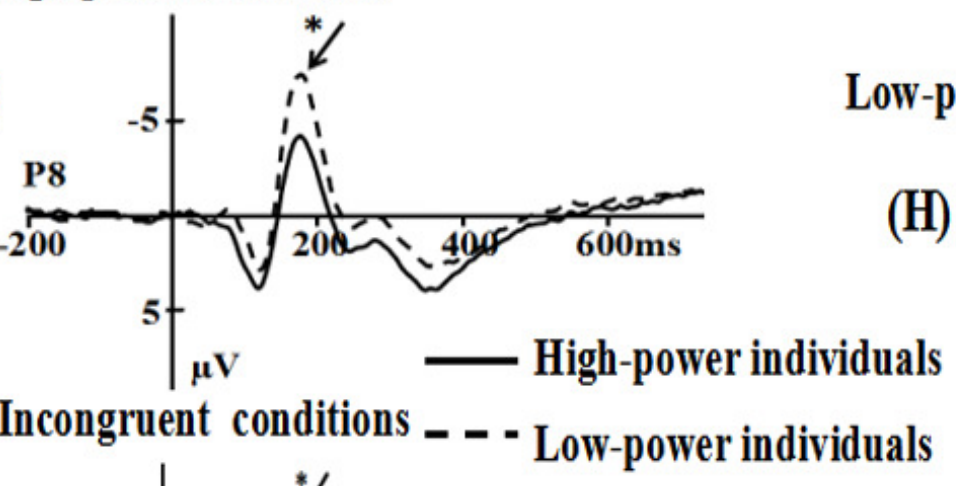

(J)

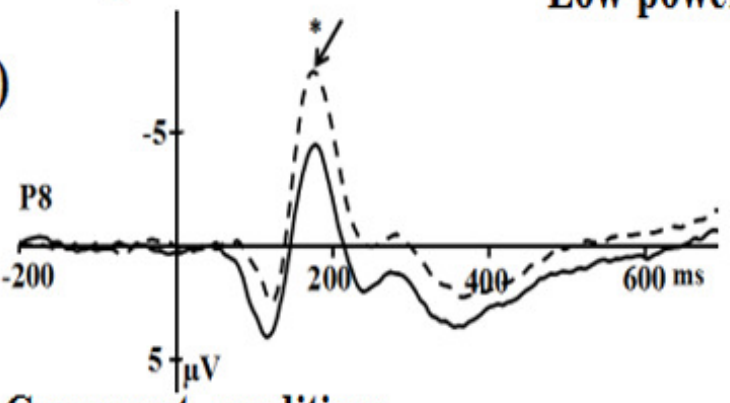

(H)

(K)
(E)

(B)

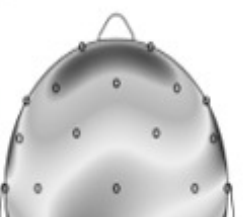

P1(120-170ms)

Incongruent conditions Congruent conditions

(C)

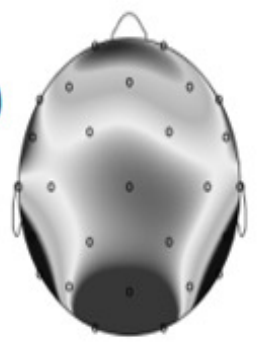

(F)

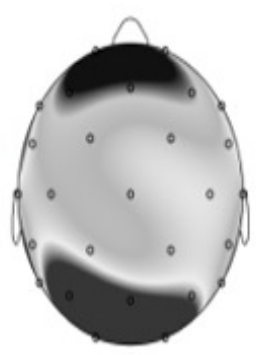

N170(150-180ms)

Low-power individuals High-power individuals
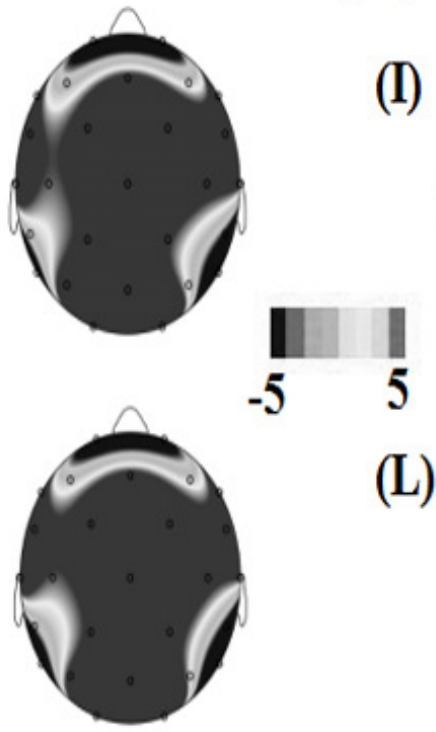

(I)

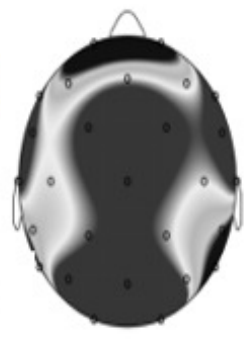

L)

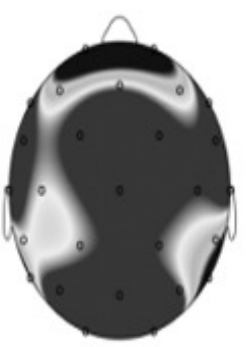




\section{Figure 6}

Waveforms and topographical maps of N170 and N450 components for fear faces.

(A) The Grand-average N170 in low-power individuals at P7 electrode site for fear faces, and

(B) Topographical maps of the N170 in incongruent conditions and (C) in congruent conditions for low-power individuals. (D) The Grand-average N170 in high-power individuals at P7 electrode site for fear faces, and (E) Topographical maps of the N170 in incongruent conditions and (F) in congruent conditions for high-power individuals. (G) The Grand-average N450 in low-power individuals at CZ electrode site for fear faces, and (H) Topographical maps of the N450 in incongruent conditions and (I) in congruent conditions for low-power individuals. (J) The Grand-average N450 in high-power individuals at CZ electrode site for fear faces, and (K) Topographical maps of the N450 in incongruent conditions and (L) in congruent conditions for high-power individuals. 

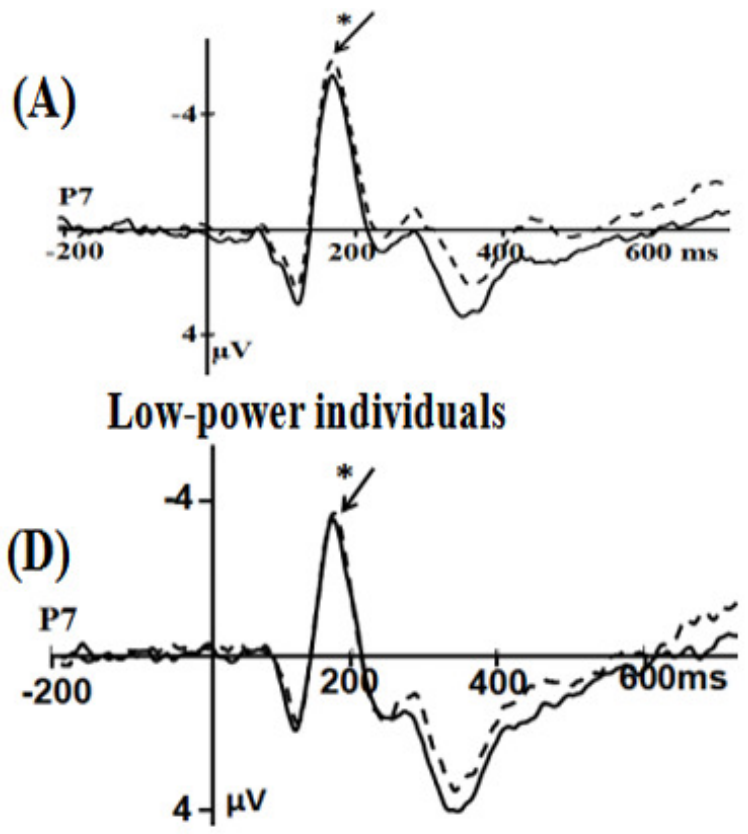

\author{
$\mathrm{N} 170(150-180 \mathrm{~ms})$ \\ Incongruent conditions Congruent conditions
}

(B)

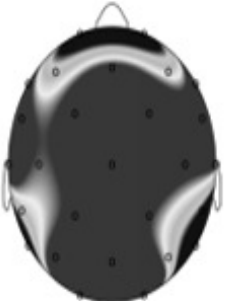

(E)

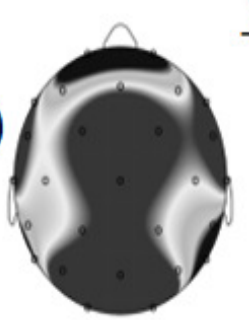

(C)

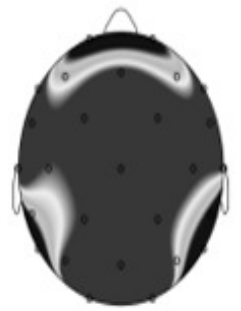

(F)

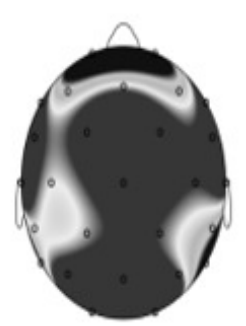

High-power individuals
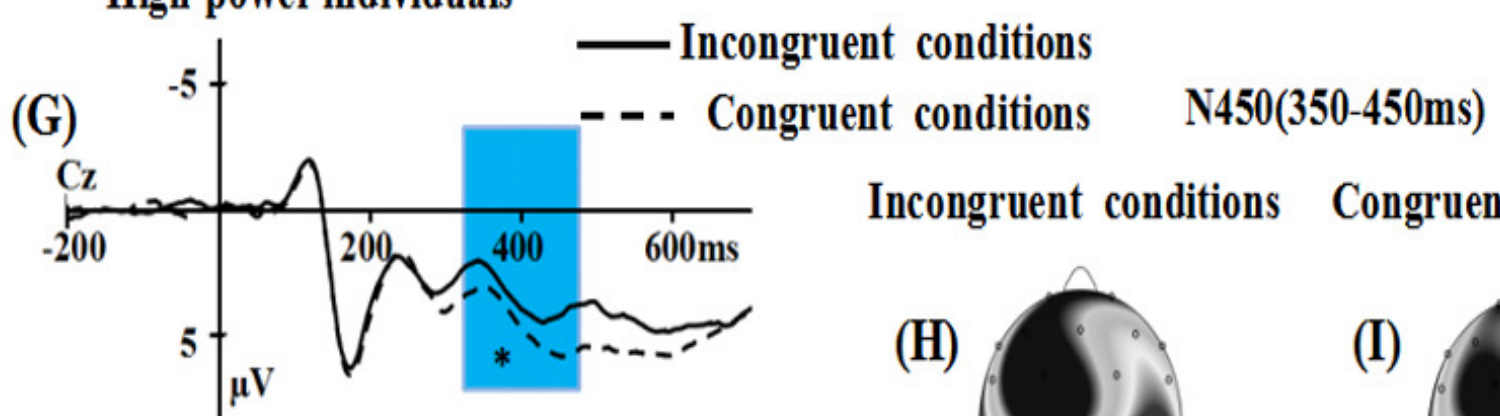

Low-power individuals

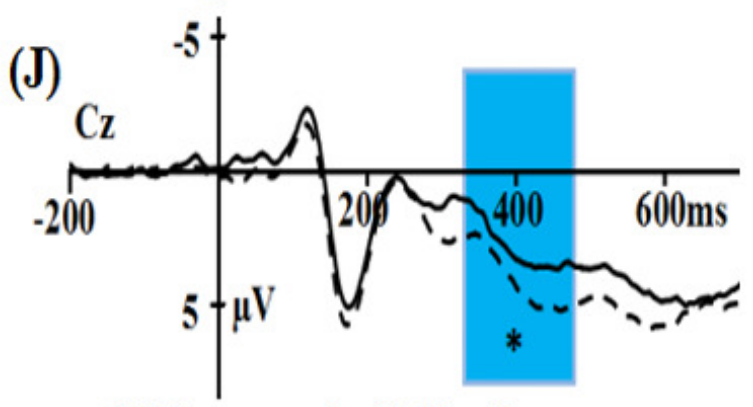

High-power individuals

Incongruent conditions Congruent conditions

(H)

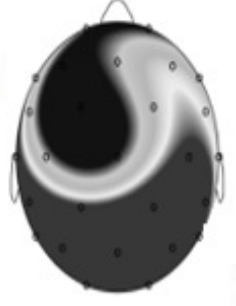

(K)

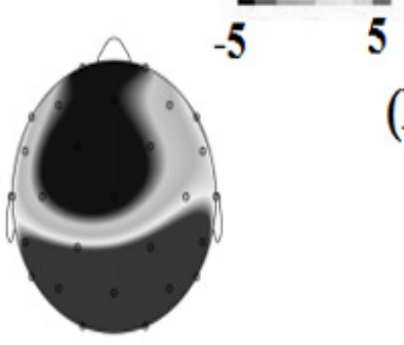

(I)

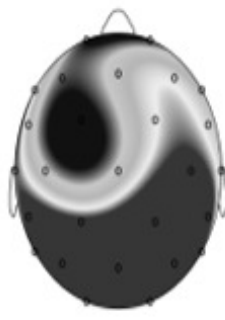

(L)

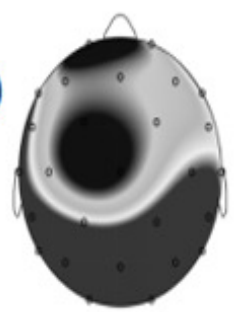




\section{Figure 7}

Waveforms and topographical maps of P1 and N170 components for happy faces.

(A) The Grand-average P1 in incongruent conditions at P8 electrode site for happy faces, and

(B) Topographical maps of the P1 for low-power individuals and (C) for high-power individuals in incongruent conditions. (D) The Grand-average P1 in congruent conditions at P8 electrode site for happy faces, and (E) Topographical maps of the P1 for low-power individuals and (F) for high-power individuals in congruent conditions. (G) The Grand-average N170 in incongruent conditions at P8 electrode site for happy faces, and (H) Topographical maps of the N170 for low-power individuals and (I) for high-power individuals in incongruent conditions. (J) The Grand-average N170 in congruent conditions at P8 electrode site for happy faces, and (K) Topographical maps of the N170 for low-power individuals and (L) for highpower individuals in congruent conditions. 
(A)

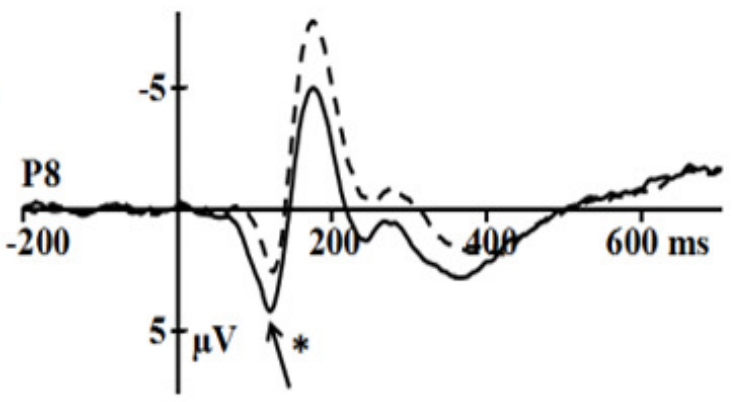

Incongruent conditions

(D)

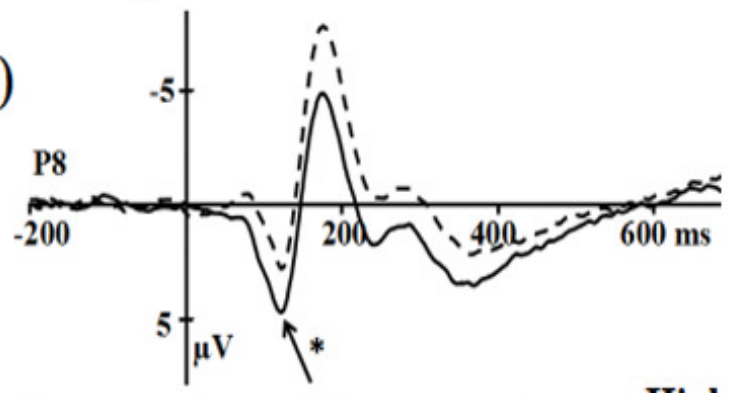

P1(120-170ms)

Low-power individuals High-power individuals

(B)

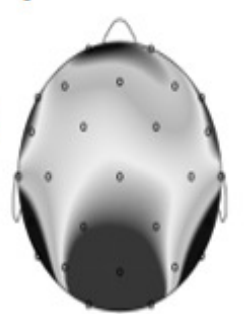

(E)

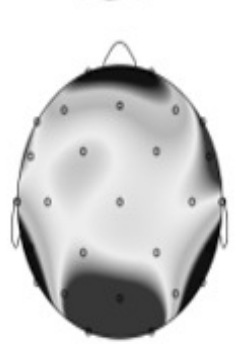

(C)

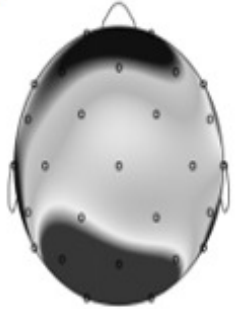

(F)

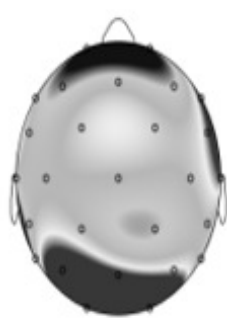

High-power individuals

(G)

Congruent conditions

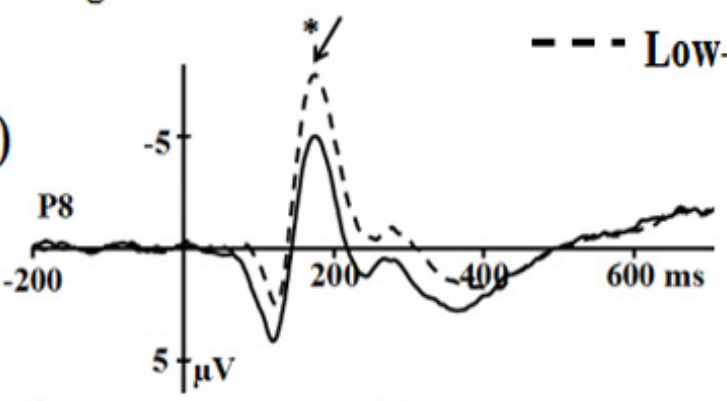

Incongruent conditions

(J)

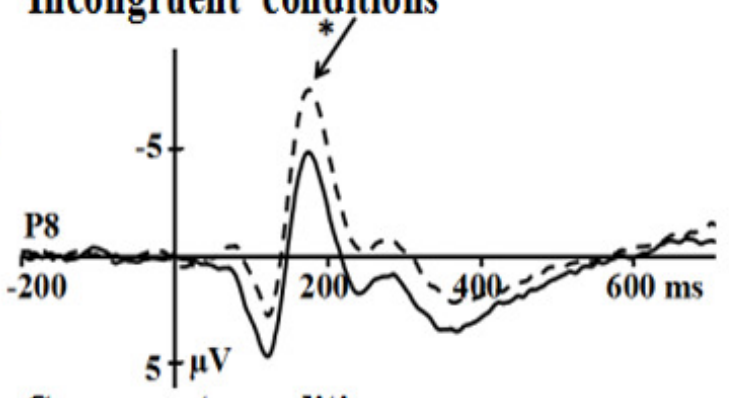

Congruent conditions

N170(150-180ms)

Low-power individuals High-power individuals

(H)

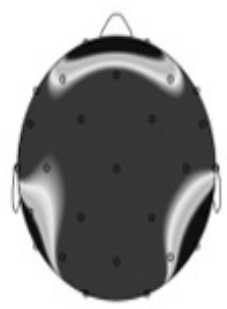

(K)

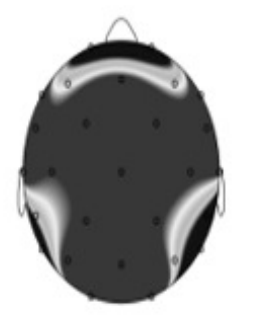

(I)

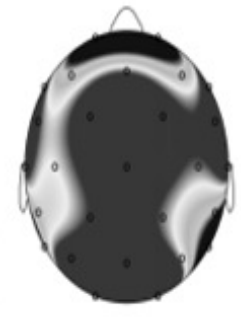

(L)

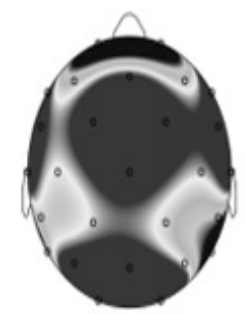


Table $\mathbf{1}$ (on next page)

The median for all conditions 
2

3 All the faces in RT

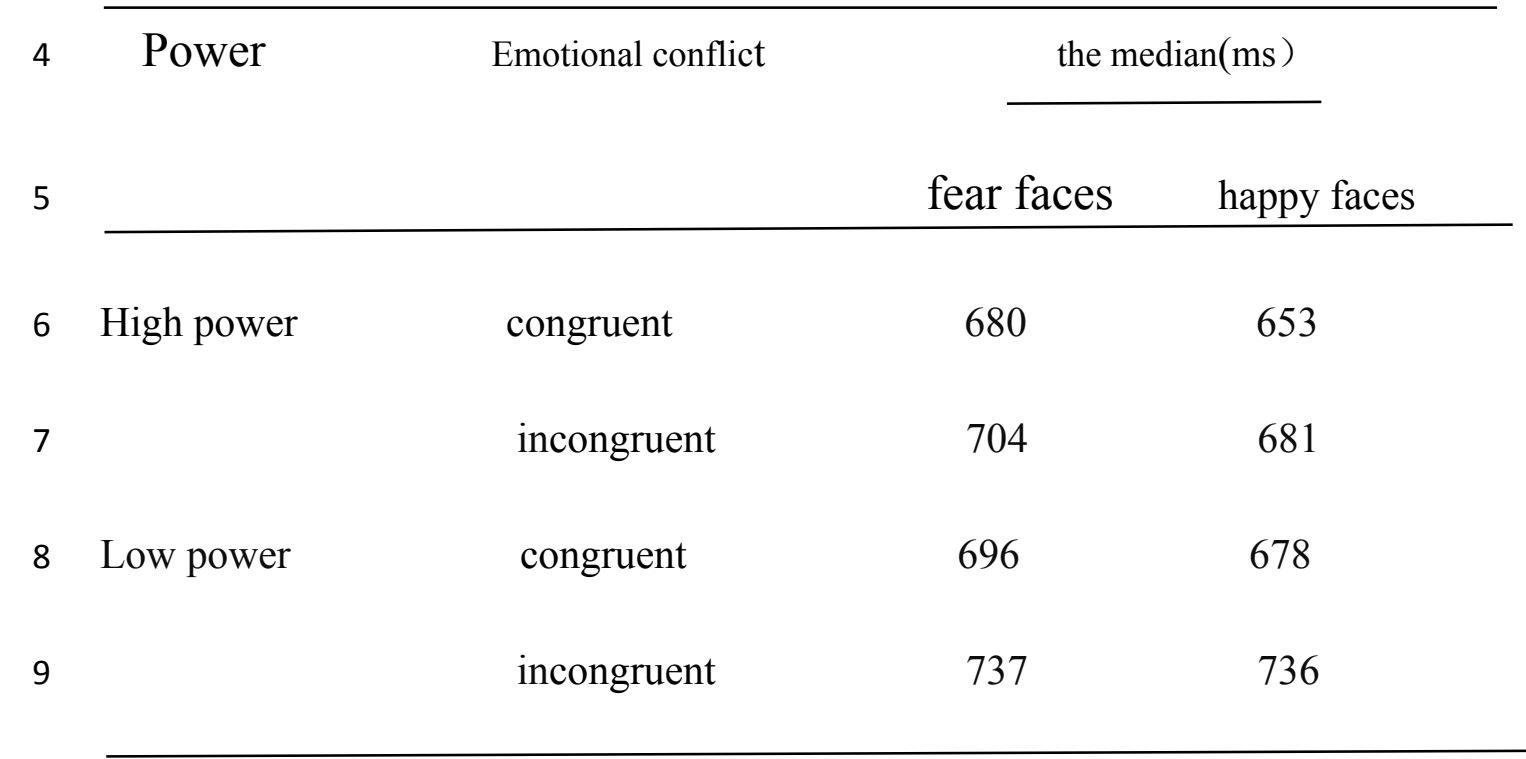

10

11

12 All the faces in ACC

13 Power

Emotional conflict

the median $(\%)$

14

fear faces happy faces

15 High power

congruent

94

95

16

incongruent

93

88

17 Low power

congruent

94

91

18

incongruent

85

81 
19

20 All the faces in P1

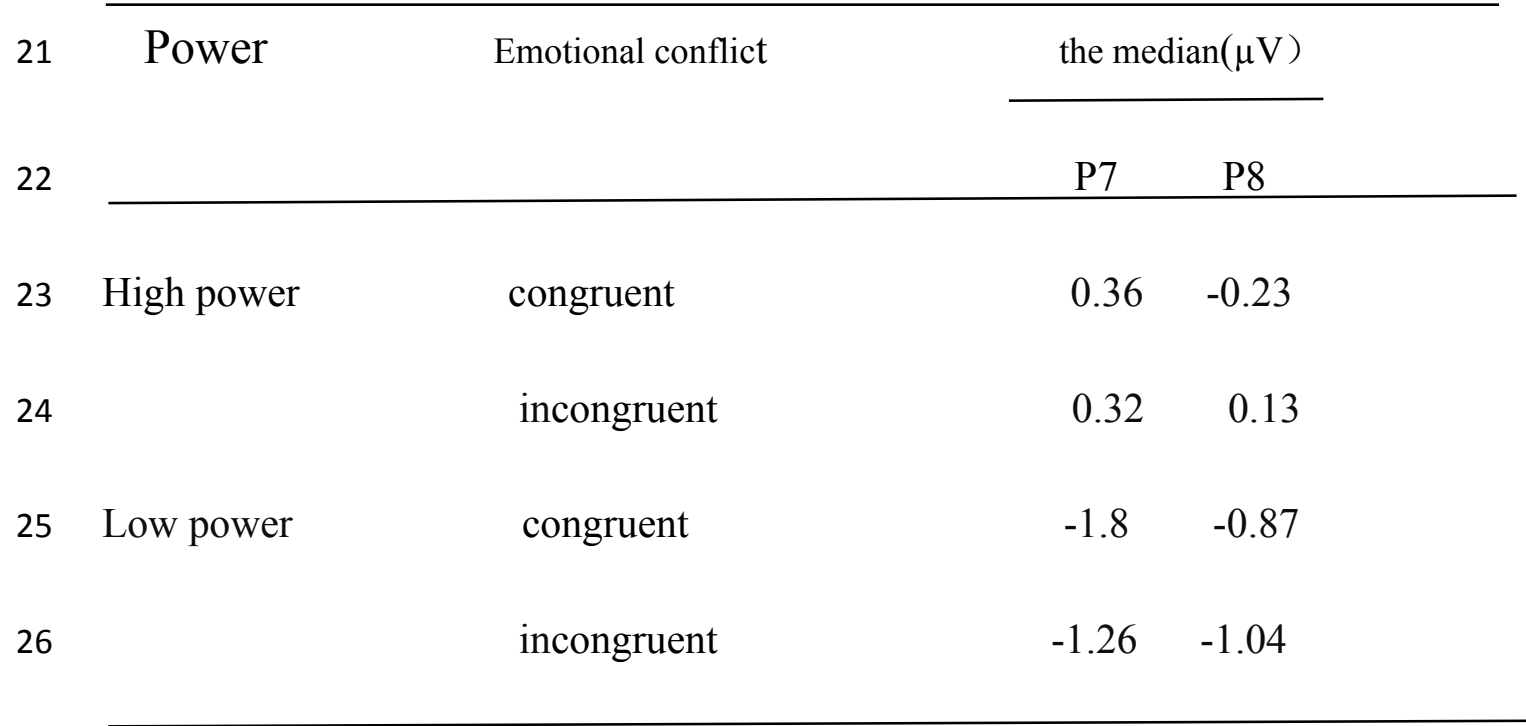

27

28

29 All the faces in N170

30

Power

Emotional conflict

the median $(\mu \mathrm{V})$

31

P7 $\quad$ P8

32

High power

congruent

$-1.41 \quad-3.57$

33

incongruent

$-1.71 \quad-3.98$

34 Low power

congruent

$-3.63 \quad-5.52$

35

incongruent

$-4.64 \quad-6.56$

36

37 
$40 \quad$ All the faces in N450

41 Power $\quad$ Emotional conflict $\quad$ the median $(\mu \mathrm{V})$

\begin{tabular}{llllllll} 
F3 & F4 & FZ & $\mathrm{C} 3$ & $\mathrm{C} 4$ & $\mathrm{CZ}$ & $\mathrm{CP} 1$ & $\mathrm{CP} 2$ \\
\hline
\end{tabular}

$$
\text { congruent }
$$$$
1.38 \quad 1.59
$$

0.92

2.99

5.16

$\begin{array}{lll}5.50 & 6.54 & 7.12\end{array}$

49 Only fearful faces in P1

\section{Power}

51

52 High power

53

54 Low power

55

56
Emotional conflict

the median $(\mu \mathrm{V})$

P7

$0.12 \quad 0.58$

incongruent

$0.56 \quad 0.36$

congruent

$-1.66-0.90$

incongruent

$0.86 \quad-1.13$ 
58 Only fearful faces in N170

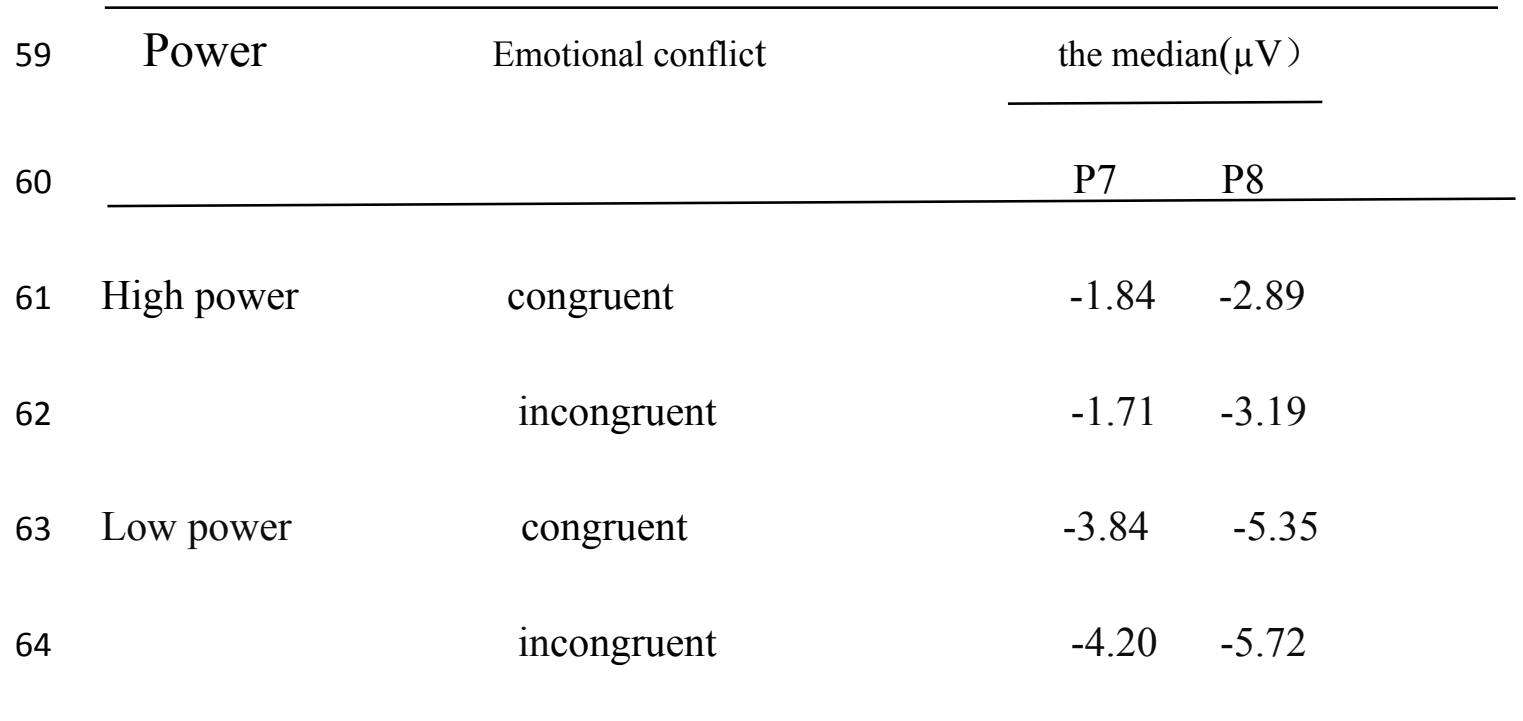

65

66

67

68 Only fearful faces in N450

69 Power Emotional conflict $\quad$ the median $(\mu \mathrm{V})$

70

\begin{tabular}{llllllll}
$\mathrm{F} 3$ & $\mathrm{~F} 4$ & $\mathrm{FZ}$ & $\mathrm{C} 3$ & $\mathrm{C} 4$ & $\mathrm{CZ}$ & $\mathrm{CP} 1$ & $\mathrm{CP} 2$ \\
\hline
\end{tabular}

71

$\begin{array}{llllllllll}\text { High power } & \text { congruent } & 1.19 & 1.47 & 1.04 & 2.94 & 4.78 & 5.66 & 6.21 & 7.09\end{array}$

72

$\begin{array}{lllllllll}\text { incongruent } & 0.63 & 1.09 & 0.40 & 1.97 & 3.51 & 3.96 & 5.19 & 6.09\end{array}$

73

Low power

congruent

$\begin{array}{llll}0.72 & 2.01 & 0.20 & 2.85\end{array}$

4.83

$\begin{array}{lll}4.39 & 5.55 & 7.08\end{array}$

74

$\begin{array}{lllllllll}\text { incongruent } & -1.16 & 2.44 & -0.10 & 2.30 & 4.58 & 3.00 & 5.47 & 6.06\end{array}$

75 
78 Only happy faces in P1

\begin{tabular}{|c|c|c|c|}
\hline \multirow[t]{2}{*}{ Power } & \multirow[t]{2}{*}{ Emotional conflict } & \multicolumn{2}{|c|}{ the median $(\mu \mathrm{V})$} \\
\hline & & P7 & $\mathrm{P} 8$ \\
\hline High power & congruent & 0.27 & -0.05 \\
\hline & incongruent & 0.44 & 0.13 \\
\hline Low power & congruent & -1.68 & -1.10 \\
\hline & incongruent & -1.35 & -1.36 \\
\hline
\end{tabular}

87 Only happy faces in N170

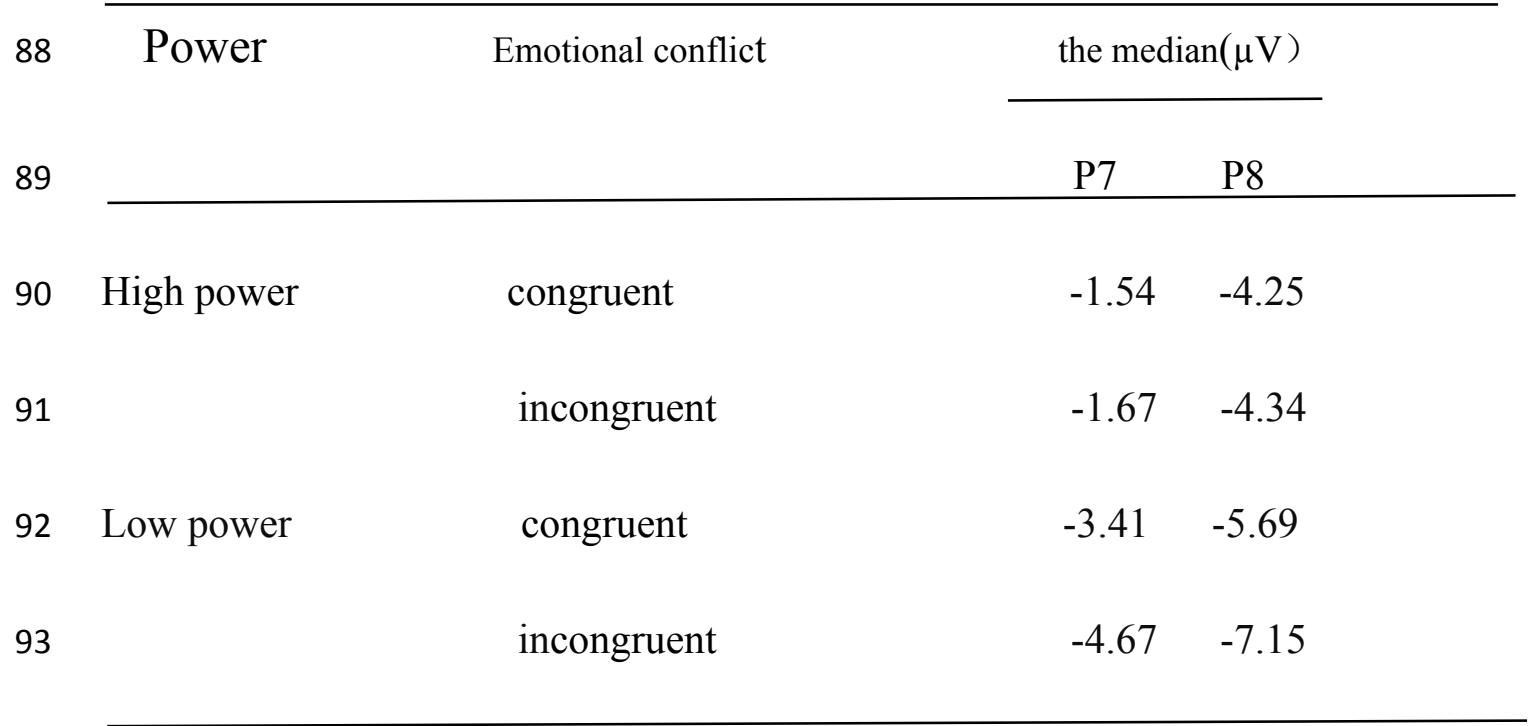


95

96 Only happy faces in N450

97 Power Emotional conflict $\quad$ the median $(\mu \mathrm{V})$

98

\begin{tabular}{llllllll} 
F3 & F4 & FZ & C3 & C4 & CZ & CP1 & CP2 \\
\hline
\end{tabular}

99

$\begin{array}{llllllllll}\text { High power } & \text { congruent } & 1.39 & 1.08 & 1.30 & 3.36 & 4.98 & 4.22 & 6.36 & 7.15\end{array}$

100

$\begin{array}{lllllllll}\text { incongruent } & 0.89 & 1.61 & 1.11 & 2.35 & 3.85 & 4.28 & 5.52 & 6.78\end{array}$

101

Low power

$\begin{array}{lllllllll}\text { congruent } & -0.81 & 1.02 & 0.24 & 2.55 & 3.86 & 3.25 & 5.60 & 6.29\end{array}$

102

$\begin{array}{lllllllll}\text { incongruent } & -0.83 & 0.67 & -0.01 & 2.17 & 3.18 & 3.46 & 5.65 & 6.26\end{array}$

103

104 
Table 2 (on next page)

Non-parametric test for all conditions 
1 Table 2 Non-parametric test for all conditions

2

3 All the faces in RT

\begin{tabular}{|c|c|c|c|}
\hline Power & Emotional conflict & $Z$ & $P$ \\
\hline Fear faces & congruent & -0.63 & 0.53 \\
\hline & incongruent & -0.95 & 0.34 \\
\hline Happy faces & congruent & -1.12 & 0.26 \\
\hline & incongruent & -1.74 & 0.08 \\
\hline
\end{tabular}

9

10

11 All the faces in ACC

\begin{tabular}{|c|c|c|c|}
\hline Power & Emotional conflict & $Z$ & $P$ \\
\hline Fear faces & congruent & -0.04 & 0.97 \\
\hline & incongruent & -0.99 & 0.32 \\
\hline Happy faces & congruent & -0.54 & 0.59 \\
\hline & incongruent & -0.34 & 0.74 \\
\hline
\end{tabular}

17

18

19 All the faces in P1 
20

21

22

23

24

25

26

27 All the faces in N170

28

29

30

31

32

33

34

35 All the faces in N450

36

37

38

\section{All the faces in $\mathrm{N} 170$}

11 the

\begin{tabular}{clcc}
\hline Electrode sites & Emotional conflict & $Z$ & $P$ \\
\hline P7 & congruent & -1.42 & 0.157 \\
& incongruent & -1.15 & 0.249 \\
P8 & congruent & -1.80 & 0.073 \\
& incongruent & -1.77 & 0.077 \\
\hline
\end{tabular}

\begin{tabular}{llcc}
\hline Electrode sites & Emotional conflict & $Z$ & $P$ \\
\hline P7 & congruent & -1.24 & 0.215 \\
& incongruent & -1.30 & 0.194 \\
& & & \\
P8 & congruent & -1.59 & 0.112 \\
& incongruent & -1.59 & 0.112 \\
\hline
\end{tabular}

\begin{tabular}{clcc}
\hline Electrode sites & Emotional conflict & $Z$ & $P$ \\
\hline F3 & congruent & -0.95 & 0.343 \\
& incongruent & -0.66 & 0.511
\end{tabular}


39

40

41

42

43

44

45

46

47

48

49

50

51

52

53

54

55

56 Only fearful faces in P1
F4

FZ

C3

C4

$\mathrm{CZ}$

CP1

$\mathrm{CP} 2$

CP2

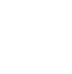

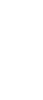

$$
\begin{aligned}
& \text { congruent } \\
& \text { incongruent }
\end{aligned}
$$

congruent

incongruent

congruent

incongruent

congruent

incongruent

congruent

incongruent

congruent

incongruent

congruent

incongruent
$-2.20$

$-0.19 \quad 0.849$

$-0.48$

$-0.42 \quad 0.672$

$-0.25 \quad 0.804$

$\begin{array}{ll}-0.34 & 0.737\end{array}$

$\begin{array}{ll}-0.25 & 0.804\end{array}$

$\begin{array}{ll}-0.31 & 0.759\end{array}$

$\begin{array}{ll}-0.10 & 0.919\end{array}$

$-0.13 \quad 0.895$

$-0.63 \quad 0.530$

$\begin{array}{ll}-1.10 & 0.919\end{array}$

$-0.02 \quad 0.988$

$\begin{array}{ll}-0.25 & 0.804\end{array}$

56 Ony

57

\begin{tabular}{llll}
\hline Electrode sites & Emotional conflict & $Z$ & $P$ \\
\hline
\end{tabular}


58

59

60

61

62

63 Only fearful faces in N170

64

65

66

67

68

69

70

71 Only fearful faces in N450

72

73

74

75

76 2
P7

congruent

incongruent

congruent

incongruent
$-1.42 \quad 0.157$

$-0.83 \quad 0.405$

$-2.03 \quad 0.042$

$-1.62 \quad 0.11$

\begin{tabular}{|c|c|c|c|}
\hline Electrode sites & Emotional conflict & $Z$ & $P$ \\
\hline P7 & congruent & -1.56 & 0.118 \\
\hline & incongruent & -1.01 & 0.314 \\
\hline P8 & congruent & -1.74 & 0.082 \\
\hline & incongruent & -1.68 & 0.093 \\
\hline
\end{tabular}

\begin{tabular}{clcc}
\hline Electrode sites & Emotional conflict & $Z$ & $P$ \\
\hline F3 & congruent & -0.45 & 0.651 \\
& incongruent & -0.37 & 0.715 \\
F4 & congruent & -0.39 & 0.693 \\
& incongruent & -0.48 & 0.630
\end{tabular}


77

78

79

80

81

82

83

84

85

86

87

88

89

90

91

92 Only happy faces in P1

93

94

95
FZ

congruent

incongruent

congruent

incongruent

C4

$\mathrm{CZ}$

CP1

$\mathrm{CP} 2$

congruent

incongruent
$-0.04 \quad 0.965$

$-0.04 \quad 0.965$

$-0.16 \quad 0.872$

$-0.13 \quad 0.895$

$-0.16 \quad 0.872$

$-0.63 \quad 0.530$

$-0.19 \quad 0.849$

$\begin{array}{ll}-0.31 & 0.759\end{array}$

$-0.51 \quad 0.609$

$-0.19 \quad 0.849$

$-0.02 \quad 0.988$

$-0.25 \quad 0.804$ 
96

97

98

99

100

101

102

103

104

105

106

107

108

109

110

111

112

113

114

07
P8

congruent

incongruent
$-1.68 \quad 0.093$

$-1.65 \quad 0.099$

Only happy faces in N170

\begin{tabular}{clcc}
\hline Electrode sites & Emotional conflict & $Z$ & $P$ \\
\hline P7 & congruent & -0.95 & 0.343 \\
& incongruent & -1.45 & 0.140 \\
& & & \\
P8 & congruent & -1.33 & 0.184 \\
& incongruent & -1.47 & 0.140 \\
\hline
\end{tabular}

5

Only happy faces in N450

\begin{tabular}{|c|c|c|c|}
\hline Electrode sites & Emotional conflict & $Z$ & $P$ \\
\hline F3 & congruent & -1.53 & 0.125 \\
\hline & incongruent & -0.92 & 0.358 \\
\hline F4 & congruent & -0.02 & 0.988 \\
\hline & incongruent & -0.10 & 0.919 \\
\hline FZ & congruent & -0.57 & 0.569 \\
\hline
\end{tabular}


115

116

117

118

119

120

121

122

123

124

125

126

127

128

129 incongruent

congruent

incongruent

C4

congruent

incongruent

congruent

incongruent

congruent

incongruent

congruent

incongruent
$-0.66 \quad 0.511$

$-0.45 \quad 0.651$

$\begin{array}{ll}-0.10 & 0.919\end{array}$

$-0.07 \quad 0.942$

$-0.04 \quad 0.965$

$-0.31 \quad 0.759$

$-0.10 \quad 0.919$

$\begin{array}{ll}-0.89 & 0.373\end{array}$

$-2.77 \quad 0.782$

$\begin{array}{ll}-0.39 & 0.693\end{array}$

$-0.16 \quad 0.872$ 